\title{
Finding the optimal nets for self-folding Kirigami
}

\author{
N. A. M. Araújo, ${ }^{1,2, *}$ R. A. da Costa, ${ }^{3}$ S. N. Dorogovtsev, ${ }^{3,4}$ and J. F. F. Mendes ${ }^{3}$ \\ ${ }^{1}$ Departamento de Física, Faculdade de Ciências, \\ Universidade de Lisboa, 1749-016 Lisboa, Portugal \\ ${ }^{2}$ Centro de Física Teórica e Computacional, Universidade de Lisboa, 1749-016 Lisboa, Portugal \\ ${ }^{3}$ Department of Physics \& $13 N$, University of Aveiro, 3810-193 Aveiro, Portugal \\ ${ }^{4}$ A. F. Ioffe Physico-Technical Institute, 194021 St. Petersburg, Russia
}

\begin{abstract}
Three-dimensional shells can be synthesized from the spontaneous self-folding of two-dimensional templates of interconnected panels, called nets. However, some nets are more likely to self-fold into the desired shell under random movements. The optimal nets are the ones that maximize the number of vertex connections, i.e., vertices that have only two of its faces cut away from each other in the net. Previous methods for finding such nets are based on random search and thus do not guarantee the optimal solution. Here, we propose a deterministic procedure. We map the connectivity of the shell into a shell graph, where the nodes and links of the graph represent the vertices and edges of the shell, respectively. Identifying the nets that maximize the number of vertex connections corresponds to finding the set of maximum leaf spanning trees of the shell graph. This method allows not only to design the self-assembly of much larger shell structures but also to apply additional design criteria, as a complete catalog of the maximum leaf spanning trees is obtained.
\end{abstract}

PACS numbers: 05.65.+b,64.60.aq

The synthesis of three-dimensional polyhedral shells at the micron and nano scales is key for encapsulation and drug delivery [1-3]. Inspired by the Japanese art of Kirigami, where hollowed structures are obtained from cutting and folding a sheet of paper, lithographic methods have been developed to form shells from twodimensional templates of interconnected panels [4-9]. The potential is enormous, for a wide range of shapes and sizes can be obtained. Ideally, the unfolded templates (nets) should spontaneously self-fold into the target structure to reduce production costs and achieve large-scale parallel production.

Many nets fold into the same structure, but some are more likely to self-fold under random movements than others. Finding the optimal net for self-folding is not trivial, as the self-folding pathway depends on the geometry of the net, its physical properties, and its interactions with the surrounding medium [10-13]. Pandey et al. combined experimental and numerical techniques to identify design principles for self-folding [14]. They have considered experimentally several nets as templates consisting of metallic hinges and panels on the submillimeter scale and let them self-fold driven by surface tension. The self-folding efficiency of each net was quantified by their yield, corresponding to the fraction of samples that selffolded into the target polyhedron, free of defects. They found that the yield is maximized when, from the entire set of nets, one picks the nets with the maximum number of so-called vertex connections (topological compactness) [14]. A vertex connection is a vertex shared in the polyhedron by two adjacent faces that share, in the net, this vertex but not an edge, see Fig. 1. Searching for this global optimum in Ref. [14] implies considering all possible nets of the shell. This inefficient procedure is time consuming, demanding, and for most shapes im-
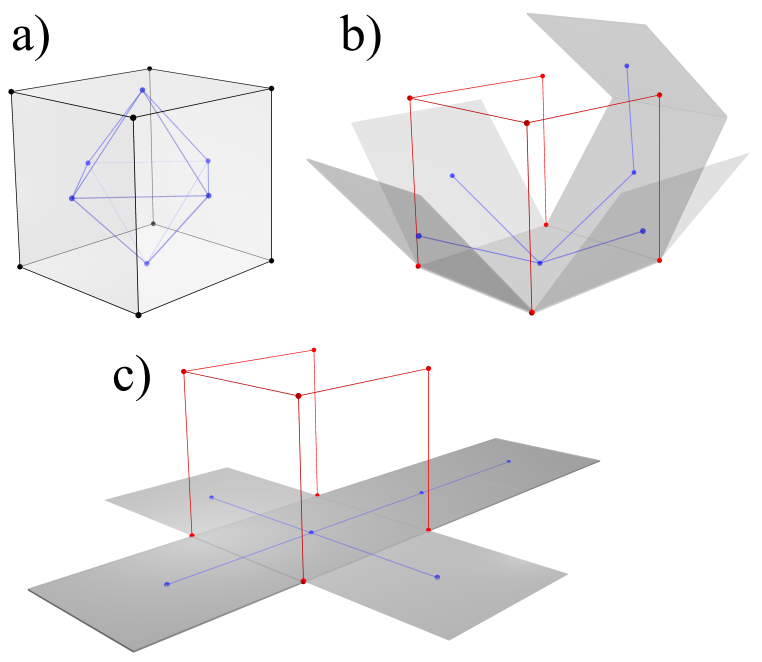

FIG. 1. Net of a cubic shell. (a) The cubic shell is mapped into a shell graph (black), where nodes and links of the shell graph are the vertices and edges of the polyhedron, respectively. In the face graph (blue), the nodes are the shell faces and the links connect pairs of adjacent faces. To unfold the shell into a two-dimensional template (net), one needs to remove a set of shell edges (e.g., red links in (b) and (c)). This set of removed shell edges is a sub-graph of the shell graph. The set of removed shell edges (cut) and the net are spanning trees of the shell and face graphs, respectively. The four vertices of the bottom face are vertex connections.

possible, since, as we show here, the total number of nets rapidly grows with the number of edges. For example, before symmetry reductions, with only twelve edges, a cube has 384 possible nets, while a dodecahedron, with thirty edges, has more than 5 million possible nets. Consequently, methods to identify optimal nets of sufficiently 
large shells have been based on random searches in the configuration space. They consider only a portion (actually a small portion) of possible nets and so they do not guarantee optimal solutions.

We propose a deterministic procedure to identify the optimal net that only requires generating a small subset of the full set of the nets of the polyhedron. Let us consider the case of a cubic shell. As shown in Fig. 1(a), the structure of the shell can be mapped into a shell graph (black nodes and links in the figure), where nodes represent the vertices and links represent the edges. A second graph can also be defined, the face graph, whose nodes are the faces and links connect pairs of adjacent faces (blue graph). Every net of the cubic shell corresponds to a spanning tree of its face graph, i.e., a connected subgraph that includes all nodes but the minimum number of links (see Fig. 1(c)). The nets can be obtained by a set of splittings along the edges of the shell graph, under the constraint that the set of nodes in the face graph remains connected. The cut is defined as the sub-graph of the shell graph that contains all removed shell edges (edges of the cut), as represented in red in Figs. 1(b) and (c). It consists of all nodes and it is a spanning tree of the shell graph. The main advantage of the mapping proposed here is that it makes possible to identify the cuts that maximize the number of vertex connections in a systematic and deterministic way, as explained below.

The vertex connections correspond to the nodes of degree one (leaves) in the cut. All cuts are spanning trees of the shell graph, so cuts with the maximum number of vertex connections correspond to maximum leaf spanning trees (MLSTs) of the shell graph. As the total number of nodes in the spanning tree is set, we can maximize the number of leaf nodes by minimizing the number of nodes connected to multiple other nodes. Thus we need to find a minimal "dominant set" of connected nodes which traverses the shell within "touching distance" (one edge) of every other node. With the addition of these edges, these other nodes are the leaves of the spanning tree, and the distance requirement ensures that they are close enough to connect to the dominant set. The algorithm we use identify the MLSTs (see Supplemental Material [15]) is not the most efficient (see, e.g. Ref. [16-18]) however it finds the full list of MLSTs while the other algorithms do not.

Figures 2(a)-(c) show three examples of threedimensional shell structures with one of their nets, corresponding to cuts that are MLSTs. For these examples, the number of spanning trees and MLSTs increase with the number of shell edges (see figure caption for values). However, the fraction of spanning trees that are MLST decays exponentially with the number of shell edges, $E$, as shown in Fig. 3. This fast decay with $E$ reinforces the necessity of a deterministic method, since the chances of obtaining a MLST from a random search is given by this fraction. For example, for the dodecahedron, with only twelve faces, less than $0.04 \%$ of its more than 5 million spanning trees are MLSTs, i.e., to obtain a MLST one would need to randomly sample 2500 configurations on average. For the largest shell that we have considered $(E=150)$, this number is larger than $10^{26}$. Thus, identifying the optimal net from random methods is practically impossible for such large shells. To explain this exponential decay of the fraction of spanning trees that are MLST, we first estimate how the number of leaves, $L$, in a MLST scales with $E$. Assuming that the shell is a convex polyhedral with regular faces and a simplified (approximated) procedure to identify the MLST, we can predict that

$$
L \sim E / 4+2,
$$

see in detail in the Supplemental Material [15]. Figure 4 shows $L$ as a function of $E$ for all Platonic and Archimedean solids with up to 150 shell edges. The obtained dependence is consistent with the predicted linear dependence (solid line). From the simple relation (1), one can estimate an upper bound for the number of MLSTs, $N_{\mathrm{MLST}}$, as a function of $E$. The exact number of spanning trees, $N_{\mathrm{ST}}$, is given by the Kirchhoff's matrix-tree theorem, which states that the total number of labeled spanning trees is given by

$$
N_{\mathrm{ST}}=\frac{1}{V} \prod_{i=1}^{V-1} \lambda_{i}
$$

where $V$ is the number of vertices, $\lambda_{n}$ the eigenvalues of the Laplacian matrix, and $\lambda_{V}=0$ is excluded from the product. The Laplacian matrix $L$ of a graph with $V$ nodes is $L=D-A$, where $D$ and $A$ are the degree and adjacency matrices, respectively. The degree matrix $D$ is a diagonal matrix where the element $D_{i i}$ is the total number of connections of node $i$. The elements of the adjacency matrix $A_{i j}$ are either one, if nodes $i$ and $j$ are connected, or zero otherwise. We can get an upper bound for $N_{\mathrm{ST}}$ as a function of $E$. Accordingly, as we show in the Supplemental Material [15], an estimate for the ratio $N_{\mathrm{MLST}} / N_{\mathrm{ST}}$ is given by

$$
N_{\mathrm{MLST}} / N_{\mathrm{ST}} \sim 2^{-E / 2+3 / 2},
$$

which is also consistent over more than 20 orders of magnitude with the observed exponential dependence shown in Fig. 3.

With the strategy proposed here, we can also consider open structures, without one or more faces, as the ones shown in Figs. 2(d) and (e). These shells might be relevant for several applications involving, for example, encapsulation, drug delivery, or key-lock mechanism [1$3,12]$. The shell graph for such structures is equivalent to the shell graph of the corresponding (closed) polyhedron; however, every cut includes all edges connecting two nodes of the missing face. So, to identify the optimal 
a)
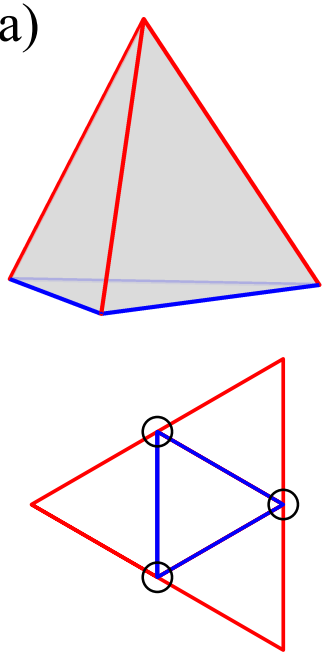

b)
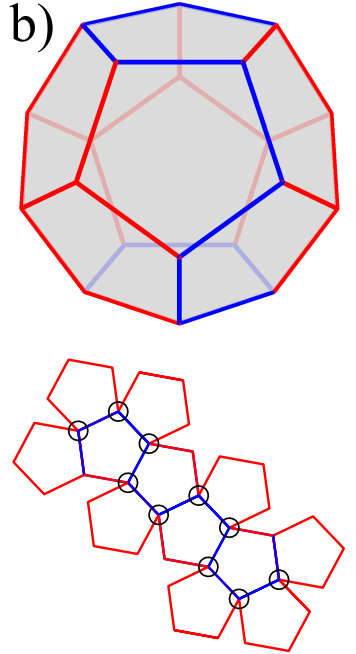

c)
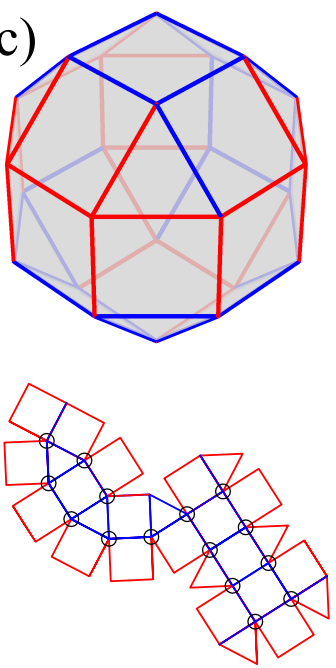

d)
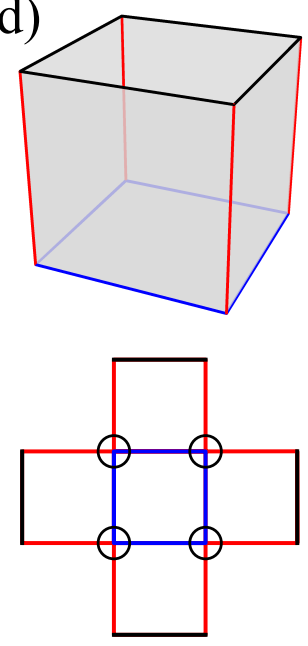

e)
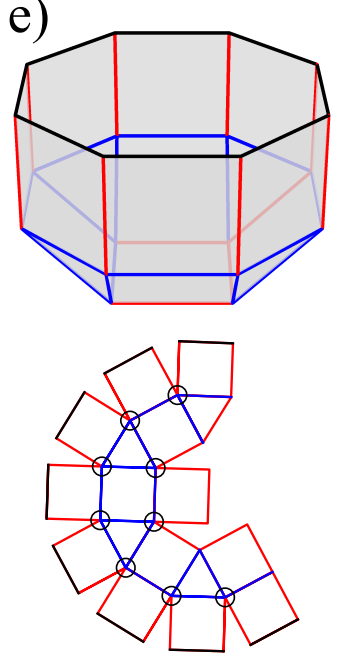

FIG. 2. Five examples of shells and of one of their nets corresponding to a cut that is a maximum leaf spanning tree: a) tetrahedron, with four faces and nine edges, it has four maximum leaf spanning trees, but only one non-isomorphic; b) dodecahedron, with twelve faces and thirty edges, it has 1980 maximum leaf spanning trees, but only 21 non-isomorphic; c) small rhombicuboctahedron, with 26 faces and 48 edges, it has 1536 maximum leaf spanning trees, but only 32 non-isomorphic; d) open cubic shell, with five faces and twelve edges, it has only one maximum leaf spanning tree e) small rhombicuboctahedron with the top nine faces removed and 17 faces, 36 edges and 20 nodes remaining, it has 720 maximum leaf spanning trees, but only 90 non-isomorphic. The black circles in the nets indicate the vertex connections.

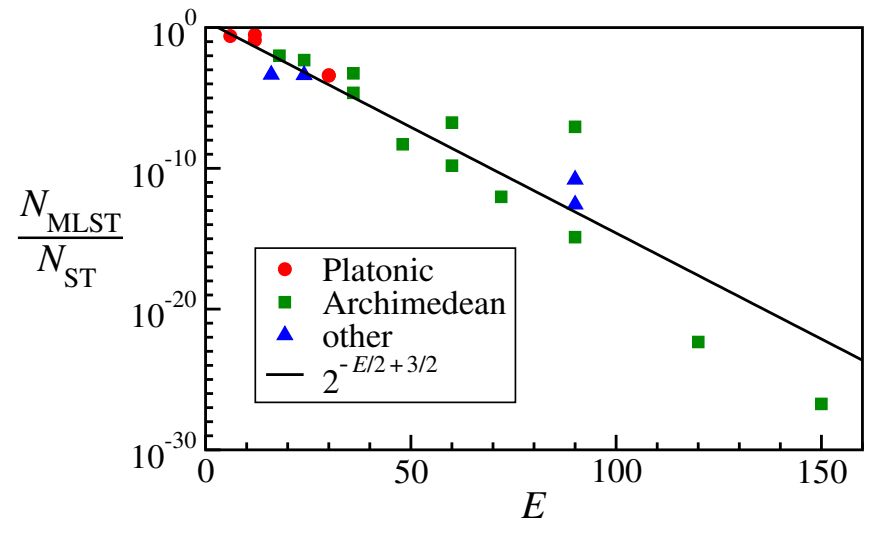

FIG. 3. Fraction of spanning trees $\left(N_{\mathrm{ST}}\right)$ that are maximum leaf spanning trees $\left(N_{\mathrm{MLST}}\right)$ as a function of the number of shell edges $(E)$. This ratio was calculated for a total of 21 shells, including all Platonic solids and all Archimedean solids with up to 150 shell edges (see Table S1 in the Supplemental Material [15]). The solid line corresponds to the estimation given by Eq. (3).

net in this case, we require that the edges of the missing face are in the cut and then follow the same procedure as before. Note that, in this case, the cut is no longer a tree, as the edges of the missing face form a loop, but this is the only possible loop in the cut as any other will split the net into pieces (see Supplemental Materials for further details [15]).

Previous studies suggested additional criteria to identify optimal structures, such as, the minimum radius of

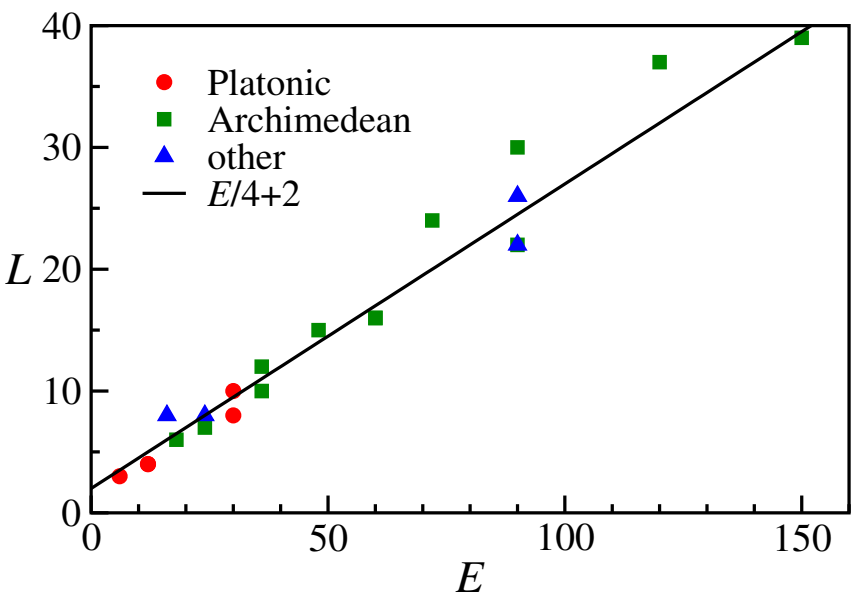

FIG. 4. Number of leaves $(L)$ as a function of the number of shell edges $(E)$. This number was calculated for a total of 21 shells, including all Platonic solids and all Archimedean solids with up to 150 shell edges (see Table S1 in the Supplemental Material [15]). The solid line corresponds to the estimation given by Eq. (1).

gyration (geometric compactness), number and type of symmetric elements, or type and shape of intermediate structures during the folding $[12,14]$. Once we find the full set of nets with the maximum number of so-called vertex connections, we can apply additional criteria to a much smaller set of nets. As an example, we will consider the additional criterion of geometric compactness where we pick the net with the lowest radius of gyration. To ap- 
a)

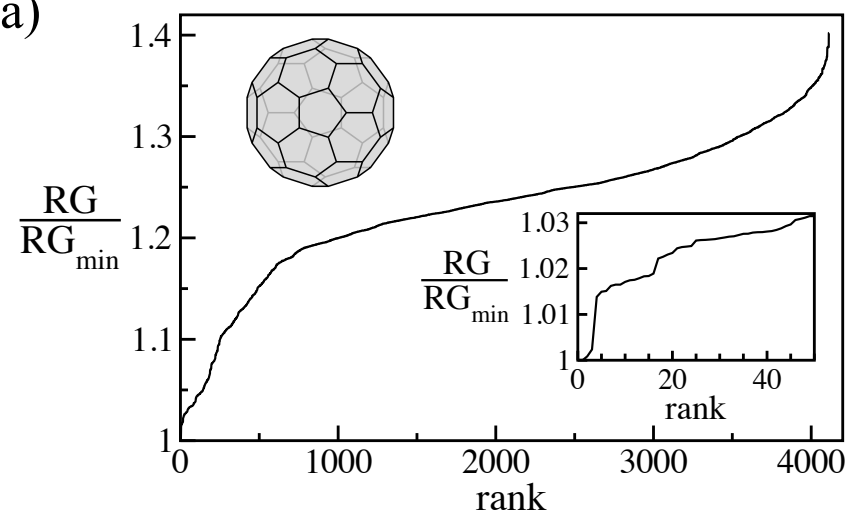

b)

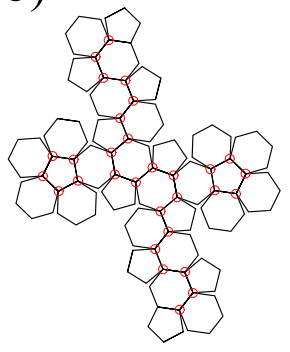

c)

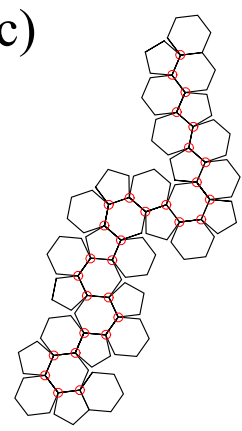

d)

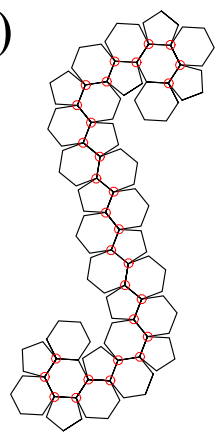

FIG. 5. For the truncated icosahedron, also known as soccer ball or buckyball: (a) spectrum of the radii of gyration for all the 4114 non-isomorphic MLSTs, where MLSTs were ordered by increasing radius of gyration; the MLST with the (b) optimal (rank 1); (c) intermediate (rank 2057: $\mathrm{RG} / \mathrm{RG}_{\min } \approx$ 1.23 ); and $(\mathrm{d})$ largest (rank $4114: \mathrm{RG} / \mathrm{RG}_{\min } \approx 1.40$ ) radius of gyration.

ply this criterion, the label of the individual nodes in the MLST is irrelevant and so, for simplicity, we first identify the subset of non-isomorphic cuts, i.e., the subset of cuts that we cannot get one from another by relabeling the nodes (or faces). This is a much smaller subset. For example, for the cubic shell, 120 MLSTs were identified, but only four are non-isomorphic. To identify them, we rely on the concept of the adjacency matrix. Two graphs are isomorphic if their adjacency matrices can be turned into each other by a sequence of line and column swaps. Note that, each swap corresponds to a relabeling of the nodes and thus the matching swaps need to be done for the lines and the columns.

Figure 5 shows the study for the truncated icosahedron, also known as soccer ball or buckyball. With 60 vertices, 32 faces, and 90 edges, this shell has more than $10^{20}$ possible cuts. By mapping the shell into a graph, we can identify the 484800 cuts with the maximum number of leaves (30 leaves each) and identify the 4114 nonisomorphic corresponding nets. To calculate the radius of gyration $R_{g}$ for each net, we first determine the centroid of the region $\alpha \subset \mathbb{R}^{2}$, corresponding to the set of

all faces of the net. The centroid is defined as

$$
(\bar{x}, \bar{y})=\frac{1}{A} \int_{\alpha}(x, y) d A,
$$

where $A$ is the total area of the shell faces. We then calculate the radius of gyration with respect to the centroid, defined as

$$
R_{g}=\sqrt{\frac{1}{A} \int_{\alpha}\left[(x-\bar{x})^{2}+(y-\bar{y})^{2}\right] d A} .
$$

To evaluate this integral, we use a standard method that only requires the coordinates of the vertices and centroid, as explained in Ref. [19]. Figure 5(a) shows the spectrum of the radii of gyration for all the 4114 nonisomorphic nets of the truncated icosahedron, ranked by increasing radius of gyration; Figures 5(b)-(c) show from those nets, the ones with the lowest (optimal), intermediate, and maximum $R_{g}$. The radius of gyration rapidly increases with the position in the rank. The less optimal net (Fig. 5(d)) has a radius of gyration that is $40 \%$ higher than the optimal net (Fig. 5(b)).

Previous methods to identify the optimal net are based on random search. Using such methods, even if the obtained net corresponds to one with the maximum number of vertex connections, the probability that its radius of gyration differs by less that $3 \%$ from the optimal one is below $1.5 \%$ (see inset in Fig. 5(a)). Since the timescale and yield of the self-folding depends strongly on the radius of gyration, the self-folding efficiency of an approximated solution obtained with previous methods based on a random search is likely far from optimal.

Conclusions. We proposed a method to identify the optimal net that spontaneously self-folds into a closed or open polyhedral shell structures. The method consists of mapping the shell structure into a shell graph and find the cuts that maximize the number of vertex connections. Adapting concepts and methods from Graph Theory, we show that the optimal solution can be obtained in a deterministic and systematic manner. Previous methods are based on random search and do not provide an optimal solution. As we showed, the fraction of nets that have the maximum number of vertex connections decays exponentially with the number of edges in the polyhedron, reinforcing the necessity of a deterministic method.

To select the optimal net for self-folding, we considered solely the topological and one particular measure of the geometric compactness (radius of gyration). The authors of Ref. [14] also discussed less-specific features to be considered for further improvement. With the method proposed here, additional design criteria for further compactness optimization can be implemented straightforwardly, since a complete list of possible nets maximizing topological compactness is obtained.

We conjectured that the nets that maximize the number of vertex connections for all convex shells are non- 
overlapping, similarly to Refs. [20-22], a necessary condition for obtaining a shell from a two-dimensional template. For all shells considered here, all optimal shells are non-overlapping but nets for concave shells are more likely to overlap. In fact, this conjecture is not needed. Once the optimal net is identified, self-overlapping can be tested. If the selected net overlaps, one should proceed through the rank of increasing radius of gyration and pick the first net that does not self-overlap. If all nets for the maximum leaf spanning trees obtained overlap, one should proceed iteratively considering spanning trees with less and less leaves until we found the first that does not overlap.

Identifying the maximum leaf spanning tree is a NPcomplete problem and so the numerical complexity will still grow rapidly with the number of shell vertices. If the number of vertices is too large for the straightforward implementation of our deterministic algorithm then, in the spirit of our approach, approximated algorithms can be used that identify spanning trees with a number of leaves that is close to the maximum $[23,24]$.

We suggest that our deterministic algorithm and its variations could be used when searching for optimal design and production of even more complex selfassembling systems.

We acknowledge financial support from the Portuguese Foundation for Science and Technology (FCT) under Contract no. UID/FIS/00618/2013 and grant SFRH/BPD/123077/2016.

*nmaraujo@fc.ul.pt

[1] R. Fernandes and D. H. Gracias, "Self-folding polymeric containers for encapsulation and delivery of drugs," Adv. Drug Deliv. Rev. 64, 1579 - 1589 (2012).

[2] J. Shim, C. Perdigou, E. R. Chen, K. Bertoldi, and P. M. Reis, "Buckling-induced encapsulation of structured elastic shells under pressure," Proc. Natl. Acad. Sci. 109, 5978-5983 (2012).

[3] M. Filippousi, T. Altantzis, G. Stefanou, M. Betsiou, D. N. Bikiaris, M. Angelakeris, E. Pavlidou, D. Zamboulis, and G. Van Tendeloo, "Polyhedral iron oxide core-shell nanoparticles in a biodegradable polymeric matrix: preparation, characterization and application in magnetic particle hyperthermia and drug delivery," RSC Adv. 3, 24367-24377 (2013).

[4] D. M. Sussman, Y. Cho, T. Castle, X. Gong, E. Jung, S. Yang, and R. D. Kamien, "Algorithmic lattice kirigami: A route to pluripotent materials," Proc. Natl. Acad. Sci. 112, 7449-7453 (2015).

[5] Y. Zhang, Z. Yan, K. Nan, D. Xiao, Y. Liu, H. Luan, H. Fu, X. Wang, Q. Yang, J. Wang, W. Ren, H. Si, F. Liu, L. Yang, H. Li, J. Wang, X. Guo, H. Luo, L. Wang, Y. Huang, and J. A. Rogers, "A mechanically driven form of kirigami as a route to $3 \mathrm{~d}$ mesostructures in micro/nanomembranes," Proc. Natl. Acad. Sci. 112, 1175711764 (2015).
[6] A. Lamoureux, K. Lee, M. Shlian, S. R. Forrest, and M. Shtein, "Dynamic kirigami structures for integrated solar tracking," Nat. Commun. 6, 8092 (2015).

[7] T. C. Shyu, P. F. Damasceno, P. M. Dodd, A. Lamoureux, L. Xu, M. Shlian, M. Shtein, S. C. Glotzer, and N. A. Kotov, "A kirigami approach to engineering elasticity in nanocomposites through patterned defects," Nat. Mater. 14, 785-789 (2015).

[8] G. P. Collins, "Science and culture: Kirigami and technology cut a fine figure, together," Proc. Natl. Acad. Sci. 113, 240-241 (2016).

[9] W. M. Jacobs and D. Frenkel, "Self-assembly of structures with addressable complexity," J. Am. Chem. Soc. 138, 2457-2467 (2016).

[10] G. M. Whitesides and B. Grzybowski, "Self-assembly at all scales," Science 295, 2418-2421 (2002).

[11] T. G. Leong, P. A. Lester, T. L. Koh, E. K. Call, and D. H. Gracias, "Surface tension-driven self-folding polyhedra," Langmuir 23, 8747-8751 (2007).

[12] A. Azam, K. E. Laflin, M. Jamal, R. Fernandes, and D. H. Gracias, "Self-folding micropatterned polymeric containers," Biomed. Microdevices 13, 51-58 (2011).

[13] A. Azam, T. G. Leong, A. M. Zarafshar, and D. H. Gracias, "Compactness determines the success of cube and octahedron self-assembly," PloS one 4, e4451 (2009).

[14] S. Pandey, M. Ewing, A. Kunas, N. Nguyen, D. H. Gracias, and G. Menon, "Algorithmic design of self-folding polyhedra," Proc. Natl. Acad. Sci. 108, 19885-19890 (2011).

[15] See Supplemental Material, which includes Refs. [1, 2, 8$10]$.

[16] H. Fernau, J. Kneis, D. Kratsch, A. Langer, M. Liedloff, D. Raible, and P. Rossmanith, "An exact algorithm for the maximum leaf spanning tree problem," Theor. Comput. Sci. 412, 6290-6302 (2011).

[17] T. Fujie, "An exact algorithm for the maximum leaf spanning tree problem," Comput. Oper. Res. 30, 1931-1944 (2003).

[18] A. Lucena, N. Maculan, and L. Simonetti, "Reformulations and solution algorithms for the maximum leaf spanning tree problem," Comput. Manag. Sci. 7, 289 $311(2010)$.

[19] C. Steger, "On the calculation of arbitrary moments of polygons," Munchen Univ., Munchen, Germany, Tech. Rep. FGBV-96-05 (1996).

[20] G. C. Shephard, "Convex polytopes with convex nets," Math. Proc. Camb. Phil. Soc. 78, 389-403 (1975).

[21] W. Schlickenrieder, Nets of Polyhedra, Ph.D. thesis, Technische Universit at Berlin (1997).

[22] E. D. Demaine and J. O'Rourke, Geometric Folding Algorithms (Cambridge University Press, Cambridge, 2007).

[23] H.-I. Lu and R. Ravi, "Approximating maximum leaf spanning trees in almost linear time," J. Algorithms 29, 132-141 (1998).

[24] R. Solis-Oba, P. Bonsma, and S. Lowski, "A 2approximation algorithm for finding a spanning tree with maximum number of leaves," Algorithmica 77, 374-388 (2017).

[1] M. R. Garey and D. S. Johnson, Computers and intractability, Vol. 29 (W. H Freeman and Company, New York, 2002).

[2] F. Rosamond, "Max leaf spanning tree," in Encyclopedia of Algorithms (Springer, 2008) pp. 1-99.

[8] F. Buekenhout and M. Parker, "The number of nets of 
the regular convex polytopes in dimension $\leq 4$," Discrete Math. 186, 69-94 (1998).

[9] D. M. Cvetković, M. Doob, and H. Sachs, Spectra of Graphs: Theory and Applications, 3rd Ed. (Wiley, New
York, 1998).

[10] J. E. Hopcroft and J.-K. Wong, "Linear time algorithm for isomorphism of planar graphs (preliminary report)," in Proc. 6th Annual ACM Symp. Theory of Computing (ACM, 1974) pp. 172-184. 


\title{
Finding the optimal nets for self-folding Kirigami - Supplemental Material
}

\author{
N. A. M. Araújo, R. A. da Costa, S. N. Dorogovtsev, and J. F. F. Mendes
}

\section{S1. ONE-TO-ONE CORRESPONDENCE BETWEEN NETS AND SPANNING TREES}

To each net of a polyhedron corresponds a cut along the edges of a spanning tree of the shell graph:

i) To unfold a polyhedral shell into a $2 \mathrm{D}$ net, the cut must reach every vertex of the shell graph, and must be connected;

ii) For the polyhedron faces to remain connected as a single component, the cut cannot contain any loops.

The only subgraphs that span to every vertex, are connected, and contain no loops are spanning trees. Therefore, maximizing the number of vertex connections of a net is equivalent to maximizing the number of leaves of a spanning tree.

\section{S2. THE MAXIMUM LEAF SPANNING TREE}

The Maximum Leaf Spanning Tree (MLST) problem has been extensively studied in the scope of graph theory and computer science [S1-S7]. It consist of finding a spanning tree with the largest possible number of leaves in a given undirected unweighted graph. Finding a MLST, or just determining the number of leaves of the MLSTs of a generic graph is a well known NP-complete problem [S1].

Here, we describe a simple (exact) algorithm to find the full set of labeled MLSTs of an arbitrary (undirected and unweighted) graph. Notice that while the algorithms for the MLST problem typically find a single optimal tree [S3-S5], our algorithm provides all possible labeled MLSTs. The number of spanning trees and of MLSTs grow both exponentially with the graph size and the computation time of an algorithm that lists all MLSTs grows at least as quickly as the number of MLSTs.

A dominating set of a graph is a subset of vertices, such that all vertices of the graph either belong to the set, or are connected to at least one vertex in the set. If, in addition, those vertices (together with the edges between them) form a connected subgraph, this set is called a connected dominating set. Clearly, the non-leaf vertices of any spanning tree form a connected dominating set. Therefore, finding the maximum number of leaves in a spanning tree is equivalent to determining the minimum size of a connected dominating set. In the rest of this section, a subtree of the graph whose vertices form a connected dominating set will be called dominating subtree. (Note that the vertices of a subtree are connected by definition.)

\section{A. Algorithm}

In order to list all MLSTs, we use a search algorithm for finding the full set of dominating subtrees with exactly $n_{S}$ vertices (i.e., subtrees with $n_{S}$ vertices that are a connected dominating set of the graph). We start by checking if there are any dominating subtrees of $n_{S}=1$ vertices, which only exist when a single vertex is connected to all the other vertices in the original graph. If there is no such tree, we iteratively increase $n_{S}$ by 1 and search again. The search stops when the set of dominating subtrees with $n_{S}$ vertices is non-empty. These minimum dominating subtrees are the interiors of the MLSTs, that is, the MLSTs without the leaf vertices and their respective edges.

To finalize the construction of the MLSTs, we attach the remaining vertices to the obtained minimum dominating subtree. These vertices are the leaves of the MLST. If every leaf vertex only has one edge of the original graph connecting it to the dominating subtree, then there is only one possible MLST with that particular interior subtree. However, some leaf vertices may have multiple edges of the original graph linking them to the dominating subtree, and so there are multiple MLSTs with the same interior subtree. In these cases, we have to chose one of the possibilities for each leaf vertex. Since those choices are independent from each other, the total number of MLSTs that share that particular interior subtree equals the product of the numbers of possibilities of each leaf vertex (i.e., the number of different ways of connecting the leaf vertices to a particular dominating subtree).

The algorithm recursively grows subtrees with $n_{S}$ vertices and $n_{S}-1$ edges of the graph starting from a single root vertex and enumerates all MLSTs where the root is a non-leaf vertex. There is a set of vertices, say $R$, that the algorithm uses as roots (one at each time), and consists of a specific arbitrary vertex and all of its neighbors. For any dominating subtree, all the vertices of the original graph must either be in the subtree or have at least one neighbor in the subtree. To find all MLSTs, it is sufficient to consider the roots in $R$. In the present work, we use the vertex with the smallest degree and its neighbors as the set of roots $R$.

Given a size of the subtrees $n_{S}$, the algorithm performs a separate search for each root vertex in $R$. So, we need an additional constraint to avoid multiple counts of the MLSTs that include more than one non-leaf vertices in $R$. We introduce a set of vertices, $V_{\text {excl }}$, that are explicitly forbidden from joining the dominating subtree. In the first search, rooted in the first vertex of $R$, say $r_{1}$, the set 
$V_{\text {excl }}$ is empty and the algorithm enumerates all MLSTs where $r_{1}$ is a non-leaf vertex. Since the vertex $r_{1}$ must be a leaf in all the other MLSTs still not found, we add $r_{1}$ to $V_{\text {excl }}$. In the second search, rooted in $r_{2}$, the vertex $r_{1}$ is never included in dominating subtree, insuring that the algorithm only returns the MLSTs where $r_{1}$ is a leaf and $r_{2}$ is a non-leaf vertex. Then, $r_{2}$ is added to $V_{\text {excl }}$, the third search is made, and so on.

Given a graph with $V$ vertices labeled $i=1, \ldots, V$, let us denote the edge that connects the vertices $i$ and $j$ by $e_{i j}$. In the following Algorithms 1-3 the set of vertices that are connected to $i$ in the original graph is denoted $A_{i}$, and the set $A=\left\{A_{i}: i=1, . ., V\right\}$ provides complete information about the graph.

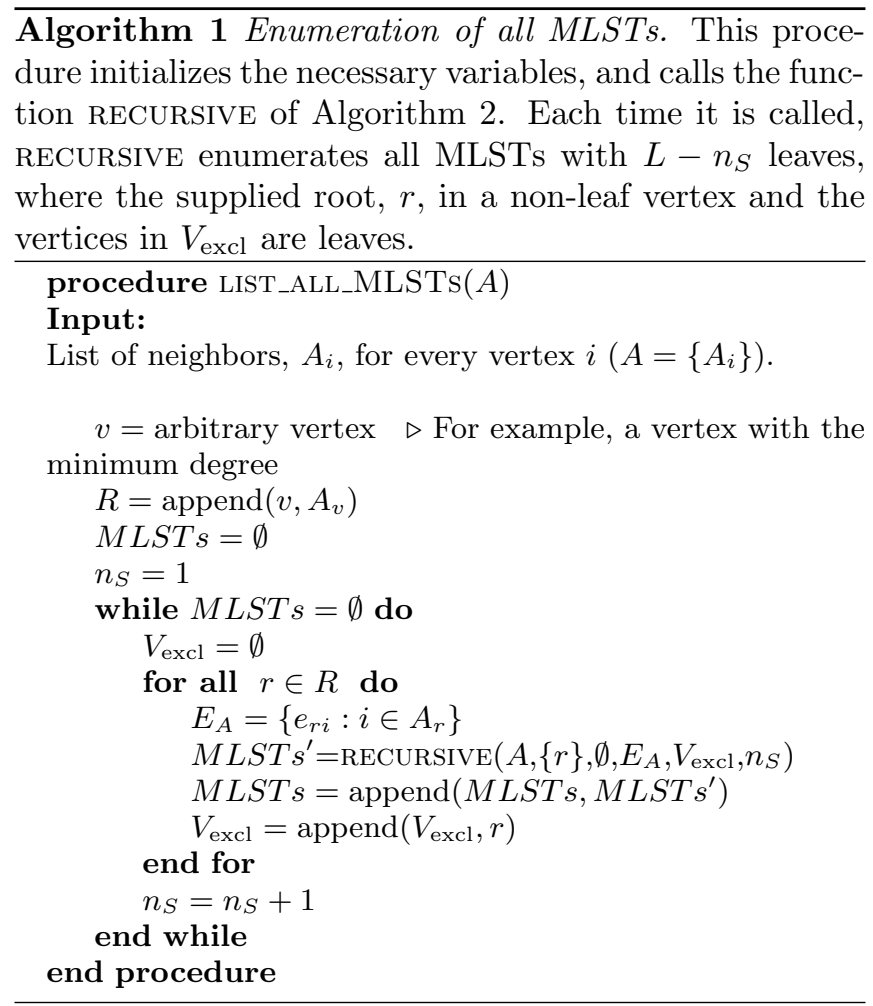

The procedure LIST_ALL_MLSTs, shown in Algorithm 1, initializes the set of roots $R$ as described above, and the current size of the searched subtrees, $n_{S}$, is initialized to 1 . The set MLSTs will store the collection of found MLSTs, and starts as an empty set. The search of MLSTs with larger $n_{S}$, and a smaller number of leaves $L=V-n_{S}$, will only proceed if the set MLSTs remains empty. The list of vertices $V_{\text {excl }}$ stores the roots of $R$ that were already used for each particular value of $n_{S}$, and is initialized as an empty set every time that $n_{S}$ is incremented. The search itself, is performed by the recursive function RECURsive, Algorithm 2. For each considered $n_{S}$, the function RECURSIVE is called by the procedure LIST_ALL_MLSTS once for each root in $R$.

The function RECURSIVE of Algorithm 2 starts with a single vertex (root) and recursively grows subtrees $T$ up to a predetermined size ( $n_{S}$ vertices), while keeping track of the elements already in the tree and at its border. Let $V_{T}$ and $E_{T}$ be the lists of vertices and edges, respectively, currently in $T$, and $E_{A}$ be the list of edges that are not in $T$ and are connected to at least one vertex in $T\left(E_{A}\right.$ is the exterior boarder of $T$, which the algorithm is currently exploring). Furthermore, $V_{\text {excl }}$ is a specific set of vertices that are forbidden to participate in the subtree $T$ (these vertices are the roots of the previous searches for the same $\left.n_{S}\right)$. When $T$ reaches the target size ( $n_{S}$ vertices) it stops increasing. If the vertices $V_{T}$ form a dominating set, then the algorithm enumerates the possible ways of joining each vertex outside of $V_{T}$ to one and only one vertex in $V_{T}$ by an edge. Each different way of making these last connections represents a different (labeled) spanning tree whose leaves are the vertices outside of $V_{T}$.

In the first stage, while the number of vertices in the growing subtree $\left|V_{T}\right|<n_{S}$, the function RECURSIVE considers all possibilities for the next edge addition to the subtree $T$ from the set of adjacent edges $E_{A}$. For each of those possibilities, RECURSIVE is called again with the updated $V_{T}{ }^{\prime}, E_{T}{ }^{\prime}$, and $E_{A}{ }^{\prime}$. Note that, when an edge in $E_{A}$ connects two vertices already in $V_{T}$ it cannot be added to the tree (because it would close a loop in $T$ ). In order to keep the vertices in the set $V_{\text {excl }}$ outside of $T$, the edges that lead to those vertices are not added to $E_{A}$ in the update. In this way, RECURSIVE finds all configurations of subtrees with $n_{S}$ vertices that include the root and exclude all the vertices in $V_{\text {excl }}$. In the second stage, when finally $\left|V_{T}\right|=n_{S}$, RECURSIVE checks if the vertices in $V_{T}$ form a dominating set: if so, it finishes the construction of the spanning trees by connecting the leaves in every possible way, otherwise it returns an empty set.

In the search algorithm presented here, we consider only connected sets of vertices (subtrees), and check if they are dominating. Furthermore, we only consider subtrees that include either a specific arbitrary vertex or at least one of its neighbors (if a subtree is dominating, every vertex of the graph fulfills this requirement). The combination of these two strategies drastically reduces the configurational space and computation time. 


\begin{abstract}
Algorithm 2 Recursive search function. This function generates all possible subtrees $T$ with $n_{S}$ vertices that include the root vertex and exclude all the vertices in $V_{\text {excl }}$. If $T$ is a dominating subtree, then the function lists all possible spanning trees that can be obtained by joining the remaining vertices of the graph to $T$.
\end{abstract}

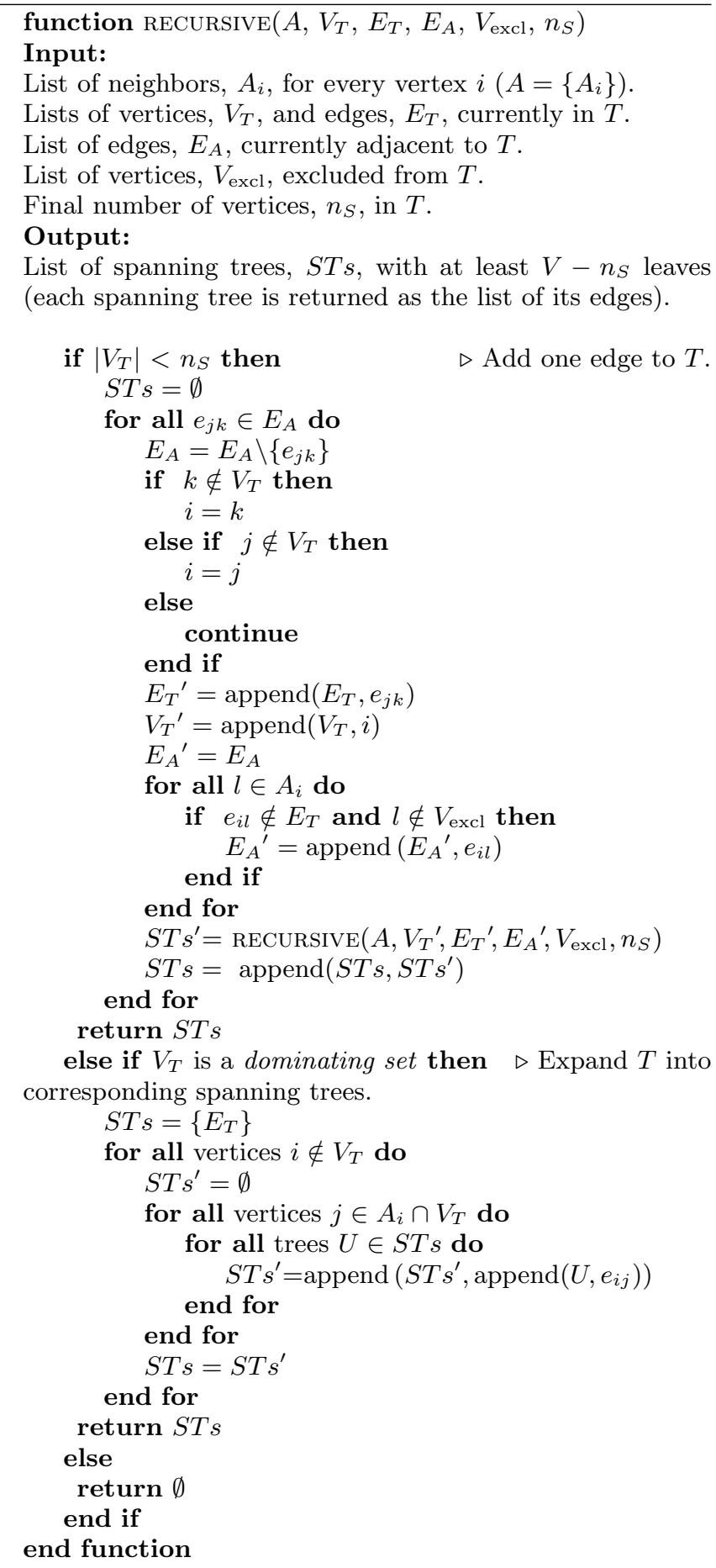

The order by which the edges of $E_{A}$ are picked in the outermost for-cycle of the search of RECURSIVE is not specified in Algorithm 2 because any order will produce the same result. In our implementation of the function
RECURSIVE, we picked the edges in $E_{A}$ using a first-infirst-out method and effectively performed a breadth-first search. If, for instance, the edges of $E_{A}$ are picked in a last-in-first-out fashion, then the algorithm will perform depth-first search instead.

The algorithm described in this section for listing all MLSTs is defined for labeled graphs, and finds the set of labeled MLSTs. If the original graph has automorphisms, i.e., a relabeling that results in the same labeled graph, then the set of labeled MLSTs may have multiple 'copies' of the same unlabeled MLST with different labelings (isomorphic MLSTs). In this work, we consider the nets of polyhedra with high regularity, such as Platonic and Archimedean solids where all vertices are equivalent. The polyhedral graphs of polyhedra where all vertices are equivalent, or with sets of equivalent vertices, have automorphisms. However, each geometrically distinct net is entirely determined by a cut of the polyhedral graph along the edges of an unlabeled spanning tree. So, to determine the number of distinct nets, we need to disregard the labels of MLSTs, and search for isomorphisms in the set of MLSTs found by the algorithm. To check if any two labeled MLSTs are isomorphic, we simply check if there is an automorphic relabeling of the original graph that maps one labeled MLST into the other, see Section for details.

Typically the algorithms for the MLST problem are designed to find the number of leaves in a MLST, and not the full set of MLSTs [S2-S5]. Some of these algorithms include multiple stages of optimization, which include heuristics, the use of approximated algorithms to make initial guesses, and other sophisticated approaches. Due to the complexity of this problem, and to the best of our knowledge, the fastest existing algorithms can find one MLST only in graphs with up to roughly 200 vertices [S5].

In this work, we find not just one MLST, but we list also the full set of optimal nets (unlabeled MLSTs) for polyhedral graphs with up to 60 vertices. The number of different optimal nets strongly depends on the details of the polyhedral graph. For instance, the Truncated Icosahedron and the Truncated Dodecahedron each have 60 vertices, 32 edges, and 90 faces, however their numbers of optimal nets are 4114 and 3719677 167, respectively. Table S1 summarizes the exact results obtained in this work with the algorithm presented here, namely the number of leaves in a MLST and the number of optimal nets of each of the 21 polyhedron considered. Table S1 also shows a figure of the optimal net which minimizes the radius of gyration for each polyhedra.

Finally, it should be mentioned that there are several approximated algorithms that find spanning trees with a high number of leaves, close to the maximum possible. If a polyhedral graph is too large to solve by exact methods, these approximated algorithms can find a near-optimal 
solution in linear or almost linear time [S6, S7].

\section{S3. SHELLS WITH HOLES}

We consider now the problem of finding optimal nets for shells that contain holes, i.e., shells consisting of all the faces of a polyhedron except for one. The graph of the vertices and edges of the shell is the same as the polyhedral graph of the complete polyhedron. The difference is that all the edges adjacent to the missing face will be in every cut, effectively detaching that face from the rest of the net, as intended. (An edge adjacent to a face is an edge between two vertices of that face.) For this reason, a vertex adjacent to the missing face cannot be a vertex connection in the net, since it always has two edges included in the cut.

The subgraph of the cut edges in the presence of a hole is not a pure tree, because it contains a single loop formed by the edges adjacent to the hole. For the shell to unfold into a 2D net, the cut subgraph must reach all vertices (spanning) and be connected. Also, for the shell's faces to remain connected in a single component, the cut subgraph cannot have any other loop apart from one surrounding the hole. Then, the cut subgraph consists of the loop adjacent to the hole, and some loopless branches connected to it.

We use the following Algorithm 3 to maximize the number of vertex connections in nets of shells that contain holes. Algorithm 3 is a simple adaptation of Algorithm 1 that calls the same recursive function of Algorithm 2, gradually increasing the allowed number of non-leaf vertices $n_{S}$. The recursive function remains unchanged in this procedure. In this version of the algorithm, in addition to the lists of adjacencies, $A=\left\{A_{i}\right\}$, we supply the list of vertices adjacent to the hole, $V_{h}$. Instead of single root vertex, the search starts from a subgraph already containing all the vertices and edges adjacent to the hole (these vertices and edges must be present in all the cuts, optimal or not). Then, in the first call of the function RECURSIVE for each $n_{S}, V_{T}=V_{h}$ and $E_{T}$ is the set edges adjacent to the hole. The set $E_{A}$ is initialized with the edges connected to the vertices of $V_{h}$ that are not in $E_{T}$, as in Algorithm 1. For the sake of clarity, while in Algorithm 1 we denote the set of edges of the optimal cuts by $M L S T s$, in Algorithm 3 we denote it by Cuts because in the presence of holes the cuts contain a loop surrounding the hole, and they are no longer trees. When Algorithm 3 calls the function RECURSIVE, it passes an empty set as the fifth input argument. This set is the list of vertices that are forced to be leaves, called $V_{\text {excl }}$ in RECURSIVE, which we do not use in this algorithm.

\begin{tabular}{|c|c|c|c|c|c|c|}
\hline & $V$ & $F$ & $E$ & $L$ & $N_{\text {opt nets }}$ & Min RG \\
\hline Tetrahedron & 4 & 6 & 4 & 3 & 1 & \\
\hline Octahedron & 6 & 8 & 12 & 4 & 2 & \\
\hline Cube & 8 & 6 & 12 & 4 & 4 & \\
\hline Icosahedron & 12 & 20 & 30 & 8 & 21 & \\
\hline Dodecahedron & 20 & 12 & 30 & 10 & 21 & \\
\hline $\begin{array}{l}\text { Octogonal } \\
\text { Piramid }\end{array}$ & 9 & 9 & 16 & 8 & 1 & \\
\hline $\begin{array}{l}\text { Octogonal } \\
\text { Dipiramid }\end{array}$ & 10 & 16 & 24 & 7 & 3 & \\
\hline $\begin{array}{c}\text { Truncated } \\
\text { Tetrahedron }\end{array}$ & 12 & 8 & 18 & 6 & 4 & \\
\hline Cuboctahedron & 12 & 14 & 24 & 7 & 34 & \\
\hline Truncated Cube & 24 & 14 & 36 & 10 & 399 & \\
\hline Snub Cube & 24 & 38 & 60 & 16 & 600 & \\
\hline $\begin{array}{l}\text { Rhombicu- } \\
\text { boctahedron }\end{array}$ & 24 & 26 & 48 & 15 & 32 & \\
\hline $\begin{array}{l}\text { Truncated } \\
\text { Octahedron }\end{array}$ & 24 & 14 & 36 & 12 & 56 & \\
\hline Icosidodecahedron & 30 & 32 & 60 & 16 & 308928 & \\
\hline $\begin{array}{c}\text { Truncated } \\
\text { Cuboctahedron }\end{array}$ & 48 & 26 & 72 & 24 & 244 & \\
\hline $\begin{array}{l}\text { Truncated } \\
\text { Icosahedron } \\
\text { (soccer ball) }\end{array}$ & 60 & 32 & 90 & 30 & 4114 & \\
\hline $\begin{array}{c}\text { Truncated } \\
\text { Dodecahedron }\end{array}$ & 60 & 32 & 90 & 22 & 3719677167 & - \\
\hline $\begin{array}{l}\text { Rhombicosi- } \\
\text { dodecahedron }\end{array}$ & 60 & 62 & 120 & 37 & 77952 & - \\
\hline $\begin{array}{c}\text { Snub } \\
\text { Dodecahedron }\end{array}$ & 60 & 92 & 150 & 39 & 13436928 & - \\
\hline $\begin{array}{c}\text { Triakis } \\
\text { Icosahedron }\end{array}$ & 32 & 60 & 90 & 26 & 664128 & - \\
\hline $\begin{array}{c}\text { Pentakis } \\
\text { Dodecahedron }\end{array}$ & 32 & 60 & 90 & 22 & 845280 & - \\
\hline
\end{tabular}

TABLE S1. Optimal nets. The number of leaves, $L$, in the MLSTs, and the number of distinct optimal nets (unlabeled

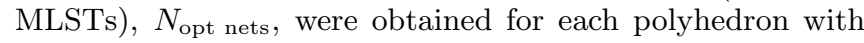
our algorithm. The numbers of vertices $V$, faces $F$, and edges $E$ are also shown, as well as the optimal net with the smallest radius of gyration (only for cases with $N_{\text {opt nets }}<10000$ ). The red circles indicate the vertex connections. 


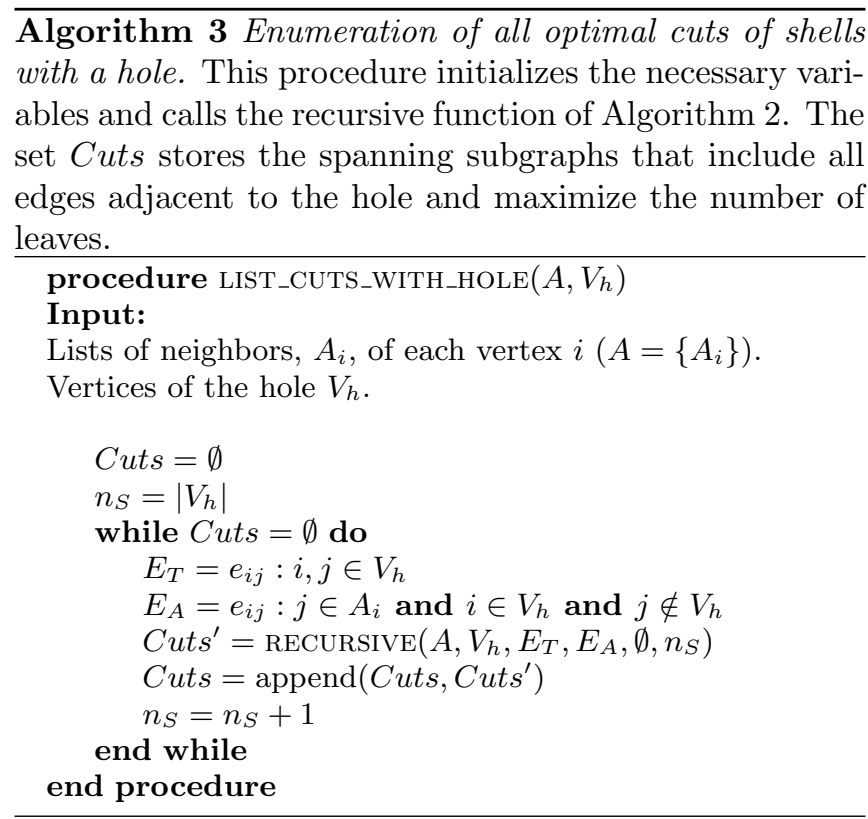

\section{S4. ESTIMATIONS}

\section{A. Maximum number of leaves}

The aim of this section is to estimate the number of leaves $L$ in a MLST in terms of the simplest possible polyhedral parameters, preferably as a function of the number of vertices $V$, or of edges $E$. The exact number $L$ depends on the details of the graph, and its determination requires to actually solve the maximum leaf spanning tree problem. However, we can obtain a simple estimate for $L$ by considering a local optimization algorithm for finding approximated maximum leaf spanning trees.

This algorithm iteratively grows a tree by progressively attaching to it vertices of the graph, until the tree becomes spanning (i.e. reaches all vertices in the polyhedral graph), in the following way:

(i) To seed the iterative process, connect the highest degree vertex to all its neighbors.

(ii) If the current tree is a spanning tree the algorithm reaches its end. Otherwise, from the vertices already in the tree, select the one with the highest number of neighbors still not in the tree, connect it to those neighbors, and repeat step (ii).

In this algorithm, except for the highest degree vertex of step (i), every vertex added to the tree starts as a leaf attached to a non-leaf vertex. The neighbors of nonleaf vertices of the intermediate (non-spanning) trees are guaranteed to be in the tree as well. Furthermore, the number of leaves in the final spanning tree is $L=V-$ $n_{\text {iter }}-1$, where $n_{\text {iter }}$ is the total number of iterations, and the -1 is due to the initial step (i). The number $n_{\text {iter }}$ depends on how many vertices are added to the tree in each iteration.
As we show in the following, the number of vertices added to the tree at each iteration turns out to be close to 2 , regardless of the details of the polyhedral (shell) graph. If the polyhedron has no triangular faces, a vertex of degree $k$ selected in step (ii) contributes at most with $k-1$ new leaves, because each vertex in the tree has at least one neighbor also in the tree. However, if the faces are triangular, any two vertices connected to each other share two common neighbors. In this case, the selected vertex has at least 3 neighbors already in the tree, namely, the parent non-leaf vertex plus two vertices that are common neighbors with the parent vertex, therefore it contributes at most with $k-3$ new leaves.

In convex polyhedra with regular faces (equilateral triangles, squares, regular pentagons, etc), the sum of the internal angles attached to a vertex must be smaller than $2 \pi$. This strongly constrains the types of faces that can be attached to a vertex of degree $k$, and in particular the number of triangles. Notice that $k$ cannot be smaller than 3 , and that $k=6$ is not feasible even with just triangles (in that case the sum of angles is equal to $2 \pi$ ). Then, let us consider the number of new vertices added to the tree when the vertex selected in step (ii) has degree $k=3,4$, and 5 . A vertex with $k=5$ is mainly surrounded by triangles (at least 4 out of 5 faces must be triangles), so, when selected in step (ii), most of the times it can contribute with $k-3=2$ new leaves to the tree. A vertex with $k=4$ must have between 1 and 4 triangles among its faces, and it can contribute with 1 to 3 new leaves depending on the particular configuration of the faces. Finally, a vertex with $k=3$ can have any number of triangles between 0 and 3 . On the one hand, if it has 3 triangles, this vertex is never selected in step (ii) because it has $0=k-3$ neighbors still not in the tree. On the other hand, if the vertex has no triangular faces attached, it can contribute with $k-1=2$ new leaves.

Due to these geometrical constraints, the average number of new vertices added to the tree at each iteration is essentially independent of the size and local details of the polyhedral graph, and is close to 2. Furthermore, we expect these arguments to qualitatively hold also for irregular convex polyhedra. Even when the internal angles of a face are different from each other, their sum is the same, and so, on average, the internal angles are the same as for a regular face.

Assuming that each iteration adds approximately 2 new vertices on average to the growing tree, and that the tree becomes spanning after $n_{\text {iter }}$ iterations, we can write:

$$
k_{0}+1+2 n_{\text {iter }} \sim V
$$

where $k_{0}$ is the highest degree in the polyhedral graph. For simplicity, let us use $k_{0}=4$. Replacing $n_{\text {iter }}=V-$ 
$L-1$ in Eq. (S1) we get

$$
L \sim(V+3) / 2
$$

This formula fits well with the general trend observed for $L$ vs. $V$, as shown in the inset of Fig. S1.

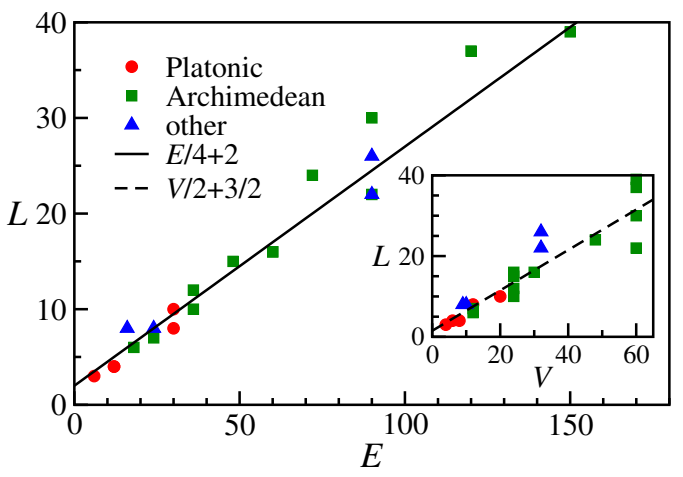

FIG. S1. Number of leaf vertices in a maximum leaf spanning tree, $L$, vs.the number of edges $E$ of the polyhedra in Table S1. Different symbols represent different sets of polyhedra. The solid line is the estimation provided by Eq (S4). The inset shows $L$ vs. the number of vertices $V$, the dashed line is the estimation provided by Eq (S2).

Interestingly, the main panel of Fig. S1 shows that the dispersion of the points is significantly smaller if we plot $L$ vs $E$. Let us recall Euler's polyhedron formula $V+$ $F=E+2$, where $V, F$, and $E$ are the numbers of vertices, faces, and edges of the polyhedron, respectively. For a given $E$ there are different polyhedra with different combinations of $V$ and $F$ such that $V+F=E+2$. On the one hand, if all faces are triangles we have $F=2 E / 3$ and in this case $V=E / 3+2$. On the other hand, if most faces of the polyhedron have many edges (which implies wide internal angles) most vertices are attached to only three faces, since the sum of the angles must be smaller than $2 \pi$ for convex polyhedra. Then, in this case $V \approx 2 E / 3$. Taking this into account, and because we consider a variety of polyhedra with different types of faces, we use the middle point,

$$
V \sim E / 2+1,
$$

as an estimation of the number of vertices $V$ of a polyhedron with $E$ edges. We replace $E / 2+1$ for $V$ in Eq. (S2), and finally obtain

$$
L \sim E / 4+2 .
$$

This formula, plotted in the main panel of Fig. S1 as a solid line, shows a remarkable agreement with our results.

\section{B. Ratio $N_{\mathrm{MLST}} / N_{\mathrm{ST}}$}

We now estimate the ratio $N_{\mathrm{MLST}} / N_{\mathrm{ST}}$, between the the numbers of maximum leaf spanning trees, $N_{\mathrm{MLST}}$, and of spanning trees, $N_{\mathrm{ST}}$, in terms of the number of edges, $E$, of a labeled polyhedral graph. These numbers grow very quickly with the size of the graph and are not uniquely determined by $E$ - they depend on the details of the graph. To obtain a simple estimate, we calculate upper bounds for $N_{\mathrm{ST}}$ and $N_{\mathrm{MLST}}$, and use their ratio as an estimator.

Each spanning tree has $V-1$ edges out of a total of $E$ edges. Therefore, the upper bound of the number of spanning trees $N_{\mathrm{ST}}$ is the number of possible combinations of $V-1$ edges, given by the binomial coefficient $\left(\begin{array}{c}E \\ V-1\end{array}\right)$. Similarly, each maximum leaf spanning tree has $L$ leaves of a total of $V$ vertices, and the upper bound for $N_{\text {MLST }}$ is the number of combinations of $L$ vertices, $\left(\begin{array}{l}V \\ L\end{array}\right)$.

To obtain an estimation for $N_{\mathrm{MLST}} / N_{\mathrm{ST}}$, we take the ratio of the upper bounds and replace the approximations for $L$ and $V$ of Eqs. (S2) and (S3), respectively:

$$
N_{\mathrm{MLST}} / N_{\mathrm{ST}} \sim\left(\begin{array}{c}
E / 2+1 \\
E / 4+2
\end{array}\right) /\left(\begin{array}{c}
E \\
E / 2
\end{array}\right) \sim 2^{-E / 2+3 / 2}
$$

where we used Stirling's approximation $n ! \sim$ $\sqrt{2 \pi n}(n / e)^{n}$. Figure S2 clearly shows that the ratio $N_{\mathrm{MLST}} / N_{\mathrm{ST}}$ has an exponential-like decay with the number of edges of the polyhedron. The simple form of Eq. (S5) fits well with this decay.

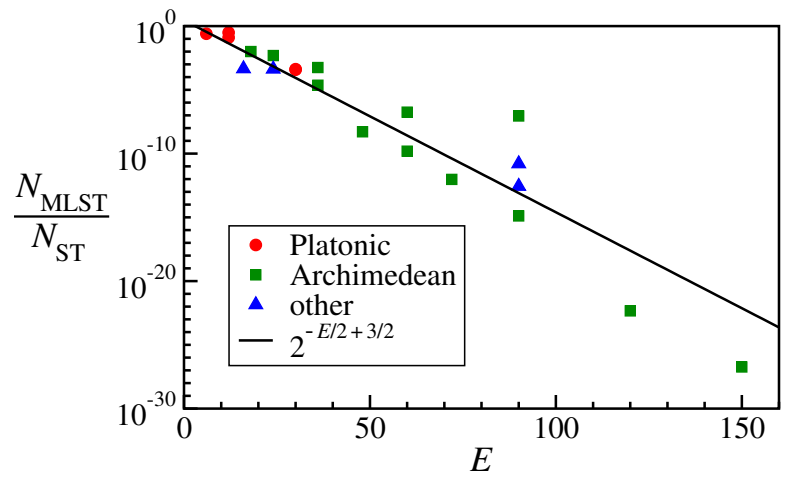

FIG. S2. Ratio between the total number of spanning trees, $N_{\mathrm{ST}}$, and the number of maximum leaf spanning trees, $N_{\mathrm{MLST}}$ vs. the number of edges $E$ of the polyhedra in Table S1. Different symbols represent different sets of polyhedra. The ratio $N_{\mathrm{MLST}} / N_{\mathrm{ST}}$ decays exponentially with the polyhedron size, as predicted by Eq. (S5).

\section{S5. NON-ISOMORPHIC CUTS}

To determine the optimal nets of a polyhedron, which have no labels, we need to find the set of distinct unlabeled MLSTs. However, the algorithm of Section distinguishes each vertex by its label, and does not consider symmetries that may exist in the polyhedral graph (automorphisms). An automorphism of a labeled graph is 
a relabeling that results in the same graph. If the polyhedral graph contains automorphisms, then the set of MLSTs may contain isomorphisms, i.e., multiple 'copies' (differently labeled) of the same unlabeled MLST [S8]. These isomorphic cuts correspond to nets that are indistinguishable from each other and have the same radius of gyration, which is our second criterion of optimization. Therefore, we need only one member of each set of isomorphic MLSTs in the list of optimal cuts. To determine if two MLSTs are isomorphic we employ an adjacency matrix approach. (Note that this approach is valid for any isomorphic subgraphs, not just MLSTs.)

The adjacency matrix, $A$, is a convenient representation of labeled graphs. Each element $A_{i j}$, is 1 if the vertices with labels $i$ and $j$ are connected by an edge, otherwise $A_{i j}$ is 0 . To switch the labels of any pair of vertices, say $i$ and $j$, we simply switch the rows $i$ and $j$ and the columns $i$ and $j$ in matrix $A$. Any permutation of $V$ labels can be mapped into any other by a series of at most $V-1$ switches between pairs of vertices ( $V$ is the number of vertices). We find the complete set of automorphisms of the polyhedral graph by comparing the relabeled adjacency matrix $A^{\prime}$ with the original matrix $A$ : when $A^{\prime}=A$ the relabeling is automorphic.

Similarly to the polyhedral graph, we can represent a MLST by an adjacency matrix $B$ where each element $B_{i j}$ is 1 if vertices $i$ and $j$ are connected by an edge in the MLST and 0 otherwise. Two MLSTs, or cuts, are isomorphic if, and only if, there is an automorphism of the polyhedral graph that maps one MLST into the other. That is, if two cuts, with adjacency matrices $B_{1}$ and $B_{2}$, unfold into the same net, then there are automorphic relabelings of $A$ that map $B_{1}$ into $B_{2}$ and vice-versa. We apply each of the previously obtained automorphisms of $A$ to one of the matrices, say $B_{2}$, and compare the relabeled matrix $B_{2}^{\prime}$ with $B_{1}$. If one of those relabeling gives $B_{2}^{\prime}=B_{1}$, then the two cuts are isomorphic, and we may discard one of them. We systematically compare each of the MLSTs remaining in the list with all the others to eliminate any isomorphisms, and obtain the full set of $N_{\text {opt net }}$ distinct optimal nets of the polyhedron.

\section{S6. NUMBER OF LABELED SPANNING TREES}

Kirchhoff's matrix-tree theorem allows us to calculate the exact number of spanning trees of a labeled graph, $N_{\mathrm{ST}}$, in terms of the spectrum of the Laplacian matrix. The Laplacian matrix of a graph is defined as $\mathcal{L}=D-A$, where $D$ is the degree matrix (i.e., the diagonal matrix with each entry $d_{i i}$ equal to the degree of vertex $i$ ), and $A$ is the adjacency matrix. For a graph of $V$ vertices the matrix $\mathcal{L}$ has $V$ eigenvalues $\lambda_{i} \geq 0$, the smallest of which is $\lambda_{V}=0$. The matrix-tree theorem states that the total number of spanning trees, $N_{\mathrm{ST}}$, is given by the product of eigenvalues of its Laplacian matrix

$$
N_{\mathrm{ST}}=\frac{1}{V} \prod_{i=1}^{V-1} \lambda_{i}
$$

where $\lambda_{V}$ is excluded from the product, and $\lambda_{i}>0$ for connected graphs and $i<V[\mathrm{~S} 8, \mathrm{~S} 9]$. The values of $N_{\mathrm{ST}}$ used to plot the points in Fig. S2 (Fig. 3 of the main text) were obtained with Eq. (S6) (while the values of $N_{\text {MLST }}$ were obtained by the algorithm described in Section ).

\begin{tabular}{c|c|c|c|c|c} 
& $V$ & $E$ & $N_{\text {nets }}$ & $N_{\mathrm{ST}} / N_{\text {aut }}$ & $\Delta$ \\
\hline Tetrahedron & 4 & 6 & 2 & $2 / 3$ & 0.66 \\
Cube & 8 & 12 & 11 & 8 & 0.27 \\
Octahedron & 6 & 12 & 11 & 8 & 0.27 \\
Icosahedron & 12 & 30 & 43380 & 43200 & 0.0041 \\
Dodecahedron & 20 & 30 & 43380 & 43200 & 0.0041 \\
5-cell & 5 & 10 & 3 & $25 / 24$ & 0.65 \\
8-cell & 16 & 32 & 261 & 216 & 0.17 \\
16-cell & 8 & 24 & 110912 & 110592 & 0.0029 \\
24-cell & 24 & 96 & $1.79 \times 10^{16}$ & $1.79 \times 10^{16}$ & $1.9 \times 10^{-11}$ \\
120-cell & 600 & 1200 & $2.76 \times 10^{119}$ & $2.76 \times 10^{119}$ & $3.9 \times 10^{-61}$ \\
600-cell & 120 & 720 & $1.20 \times 10^{307 *}$ & $1.20 \times 10^{307}$ & 0
\end{tabular}

TABLE S2. Comparison of the exact number of nets $N_{\text {nets }}$ [S8] and the estimation $N_{\mathrm{ST}} / N_{\text {aut }}$ for all regular convex polytopes in $3 \mathrm{D}$ (top 5 rows) and $4 \mathrm{D}$ (bottom 6 rows). The right-hand side column of the table is the relative difference $\Delta=\left(N_{\text {nets }}-N_{\mathrm{ST}} / N_{\text {aut }}\right) / N_{\text {nets }}$. The numbers of vertices, and edges are denoted $V$ and $E$, respectively. We include the $4 \mathrm{D}$ polytopes in this table to show that $\Delta$ approaches 0 very quickly as the size ( $V$ and $E$ ) of the polytope increases. Note that everywhere else in this paper we consider only $3 \mathrm{D}$ shells and their 2D nets.

*The number of nets of the 600-cell shown in Ref. [S8] is wrong, due to a mistake in the calculation of the graph's spectrum. Correcting the mistake gives exactly $2^{182} \cdot 3^{102} \cdot 5^{20} \cdot 7^{36} \cdot 11^{48} \cdot 23^{48} \cdot 29^{30}$ nets for the 600 -cell. In this particular case, the lower-bound and the actual number of nets coincide exactly.

\section{S7. NUMBER OF NON-ISOMORPHIC CUTS}

The number of non-isomorphic cuts (i.e. nets), $N_{\text {nets }}$, of a polyhedron with no automorphisms is equal to $N_{\mathrm{ST}}$. When the polyhedral graph has automorphisms, the number of distinct nets is actually smaller than $N_{\mathrm{ST}}$, see Section. In that case, the exact number of nets, $N_{\text {nets }}$, can be obtained using the approach of Ref. [S8], which involves a detailed, case by case, analysis for each polyhedron. In Ref. [S8] the $N_{\text {nets }}$ are obtained only for the five platonic solids. Nevertheless, we can estimate $N_{\text {nets }}$ for other polyhedron with automorphisms (e.g., Archimedean solids) with high precision by taking the ratio of $N_{\mathrm{ST}}$ to the number of automorphisms of the graph, $N_{\text {aut }}$. The ratio $N_{\mathrm{ST}} / N_{\text {aut }}$ is in fact a lower-bound of $N_{\text {nets }}$; This ratio assumes that each unlabeled spanning tree contributes with $N_{\text {aut }}$ differently labeled 'copies' to 
the set of labeled spanning trees, however, some spanning trees have a smaller number of isomorphic 'copies', due to the existence of symmetries in their branches. Since $N_{\text {aut }}$ can be found with linear time algorithms [S10], the calculation of $N_{\mathrm{ST}} / N_{\text {aut }}$ is straightforward.

Table S2 clearly shows that this lower-bound actually approaches $N_{\text {nets }}$ very quickly for large graphs. This happens because the fraction of spanning trees that have some symmetry in their structure quickly approaches 0 as the size of the graph increases. Figure S3(a) demonstrates that the number of distinct nets, $N_{\text {nets }} \approx$ $N_{\mathrm{ST}} / N_{\text {aut }}$, grows exponentially with the size of the polyhedral graph.
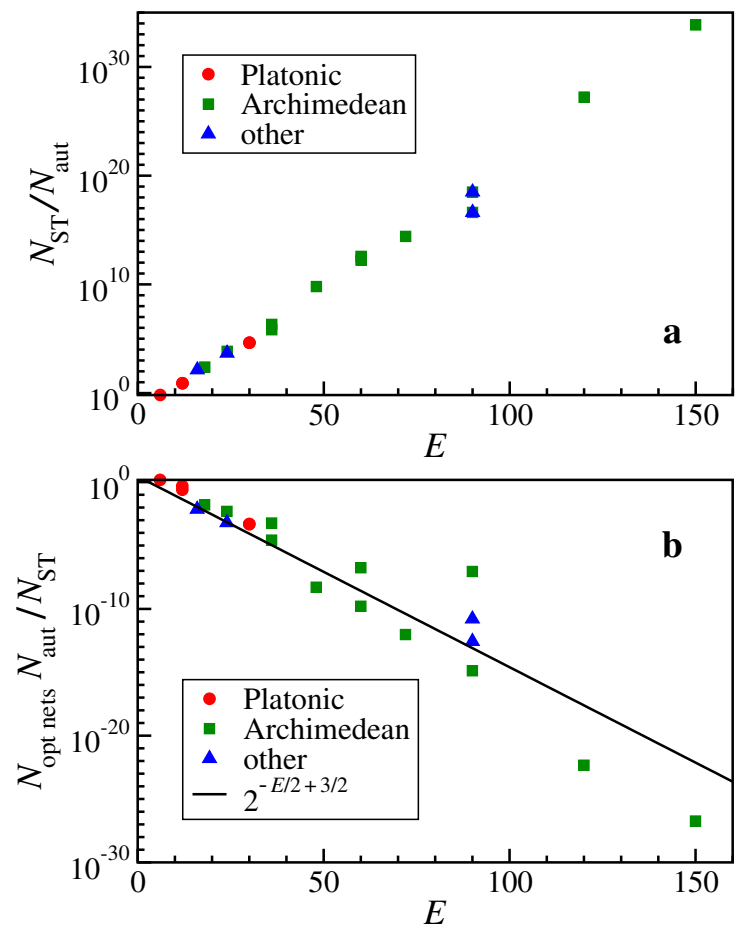

FIG. S3. Precise estimations of (a) the number of distinct nets $N_{\text {nets }} \approx N_{\mathrm{ST}} / N_{\text {aut }}$, and of (b) the fraction of optimal nets $N_{\text {opt nets }} / N_{\text {nets }} \approx N_{\text {opt nets }} N_{\text {aut }} / N_{\text {ST }}$ vs. the number of edges $E$ of the $3 \mathrm{D}$ polyhedra in Table $\mathrm{S} 1$. The number $N_{\text {nets }}$ grows exponentially with $E$, and shows a remarkably low level of dispersion. The solid line in the plot of panel (b) is Eq. (S5). The fraction of unlabeled optimal cuts $N_{\text {opt nets }} / N_{\text {nets }}$ is essentially equal to the fraction of labeled optimal cuts, $N_{\mathrm{MLST}} / N_{\mathrm{ST}}$, see Fig. S2.

The probability that a randomly sampled labeled spanning tree is a MLST is equal to the ratio $N_{\mathrm{MLST}} / N_{\mathrm{ST}}$, which is shown is Fig. 3 of the main text, and reproduced in this Supplemental Material as Fig. S2. If we were to generate nets with a method that avoids the sampling of isomorphic cuts completely, thus reducing the search space, the probability that a randomly sampled net has the maximum number of vertex connections, $N_{\text {opt nets }} / N_{\text {nets }}$, would still be essentially the same as $N_{\mathrm{MLST}} / N_{\mathrm{ST}}$.
Figure S3(b) shows the probability that a net sampled at random from the set of non-isomorphic nets has the maximum number of vertex connections, i.e., $N_{\text {opt nets }} / N_{\text {nets }} \approx N_{\text {opt nets }} N_{\text {aut }} / N_{\mathrm{ST}}$. We observe no significant differences between Figs. S2 and S3(b), which means that searches over unlabeled and labeled configurations have similar performances when only a small fraction of the configurations is sampled.

*nmaraujo@fc.ul.pt

[S1] M. R. Garey and D. S. Johnson, Computers and intractability, Vol. 29 (W. H Freeman and Company, New York, 2002).

[S2] F. Rosamond, "Max leaf spanning tree," in Encyclopedia of Algorithms (Springer, 2008) pp. 1-99.

[S3] H. Fernau, J. Kneis, D. Kratsch, A. Langer, M. Liedloff, D. Raible, and P. Rossmanith, "An exact algorithm for the maximum leaf spanning tree problem," Theor. Comput. Sci. 412, 6290-6302 (2011).

[S4] T. Fujie, "An exact algorithm for the maximum leaf spanning tree problem," Comput. Oper. Res. 30, 19311944 (2003).

[S5] A. Lucena, N. Maculan, and L. Simonetti, "Reformulations and solution algorithms for the maximum leaf spanning tree problem," Comput. Manag. Sci. 7, 289311 (2010).

[S6] R. Solis-Oba, P. Bonsma, and S. Lowski, "A 2approximation algorithm for finding a spanning tree with maximum number of leaves," Algorithmica 77, 374-388 (2017).

[S7] H.-I. Lu and R. Ravi, "Approximating maximum leaf spanning trees in almost linear time," J. Algorithms 29, 132-141 (1998).

[S8] F. Buekenhout and M. Parker, "The number of nets of the regular convex polytopes in dimension $\leq 4$," Discrete Math. 186, 69-94 (1998).

[S9] D. M. Cvetković, M. Doob, and H. Sachs, Spectra of Graphs: Theory and Applications, 3rd Ed. (Wiley, New York, 1998).

[S10] J. E. Hopcroft and J.-K. Wong, "Linear time algorithm for isomorphism of planar graphs (preliminary report)," in Proc. 6th Annual ACM Symp. Theory of Computing (ACM, 1974) pp. 172-184.

\section{S8. SOURCE CODE FOR CLOSED SHELLS}

In this sections we print the source code of a Mathematica implementation of the algorithm presented in Sec. S2. This is the code that can be found in the file Procedure1_for_closed_shells.nb available at URL https://journals.aps.org/prl/supplemental/10. 1103/PhysRevLett.120.188001. The inputs supplied to this Mathematica routine are the adjacency matrix of the shell graph, the lists of vertices adjacent to each face, and the spatial coordinates of each vertex in the polyhedron. During its execution the script outputs 
informations about the progress of the algorithm, and returns a list of all the nets with the maximum number of vertex connections. This list is sorted by the radius of gyration. The script also produces a plot of the ranked radii of gyration for the optimal nets found by the algorithm. The particular polyhedron treated by script printed here is the structure presented in Fig. 2c of the main paper, the Small Rhombicuboctahedron.

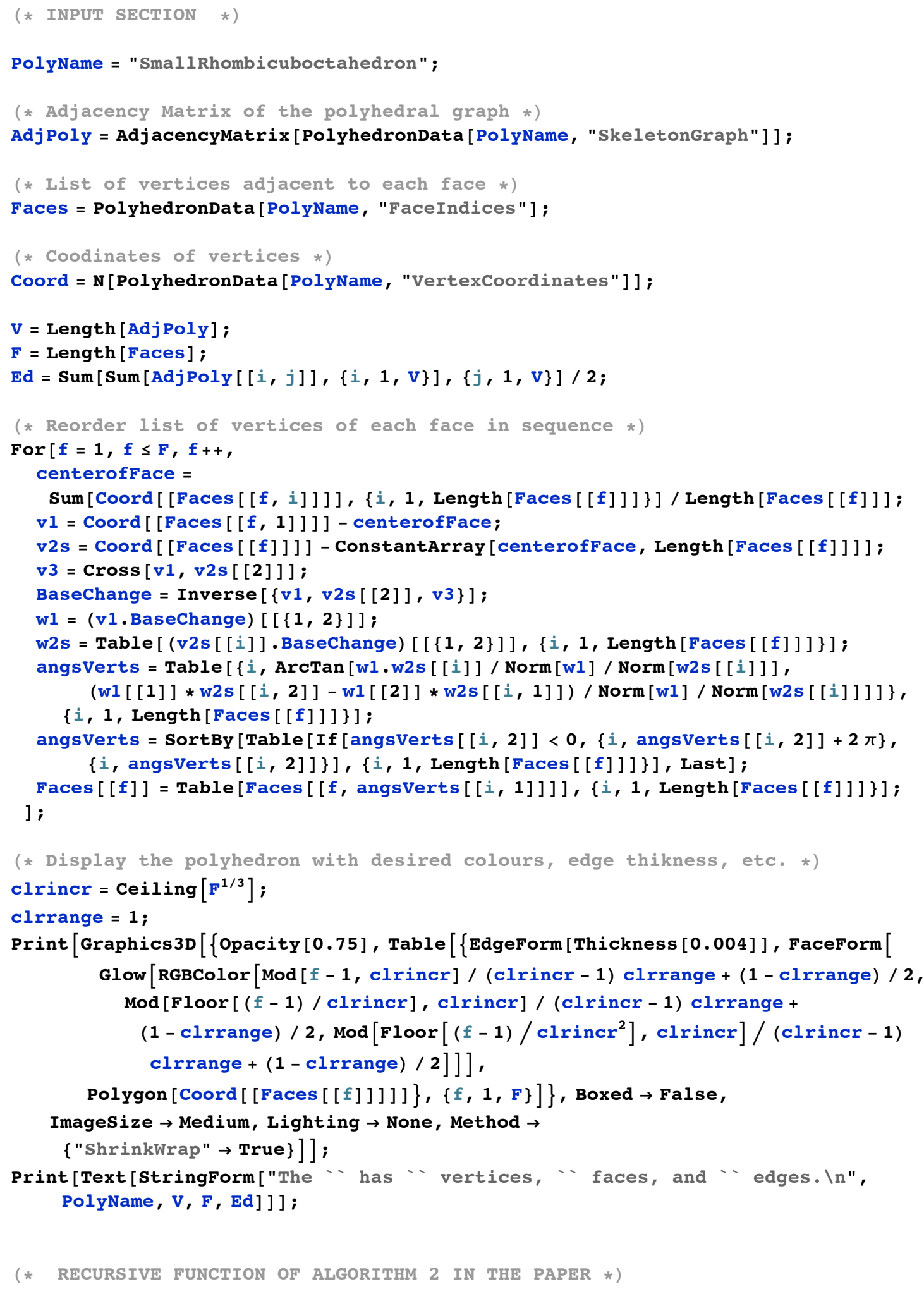




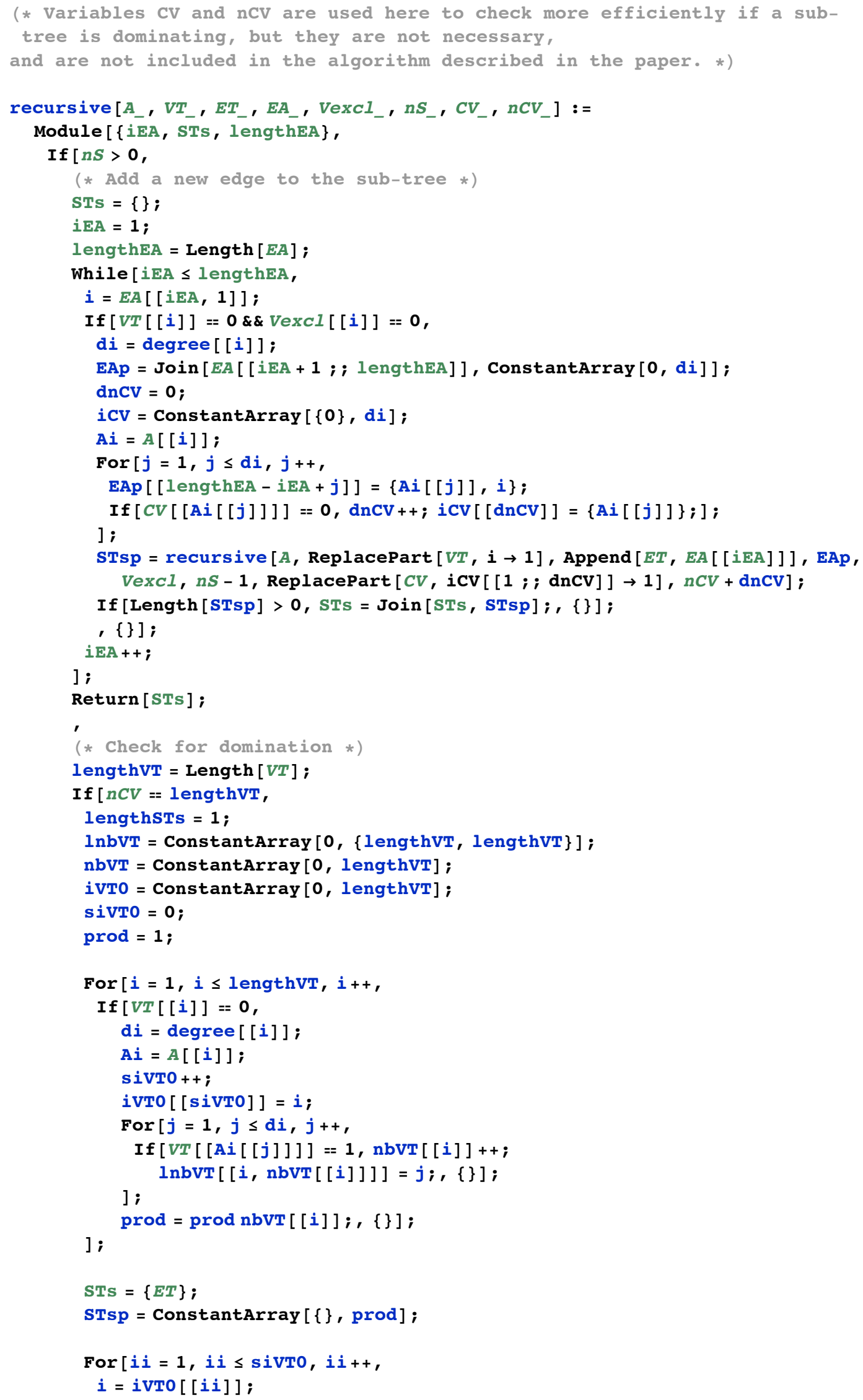




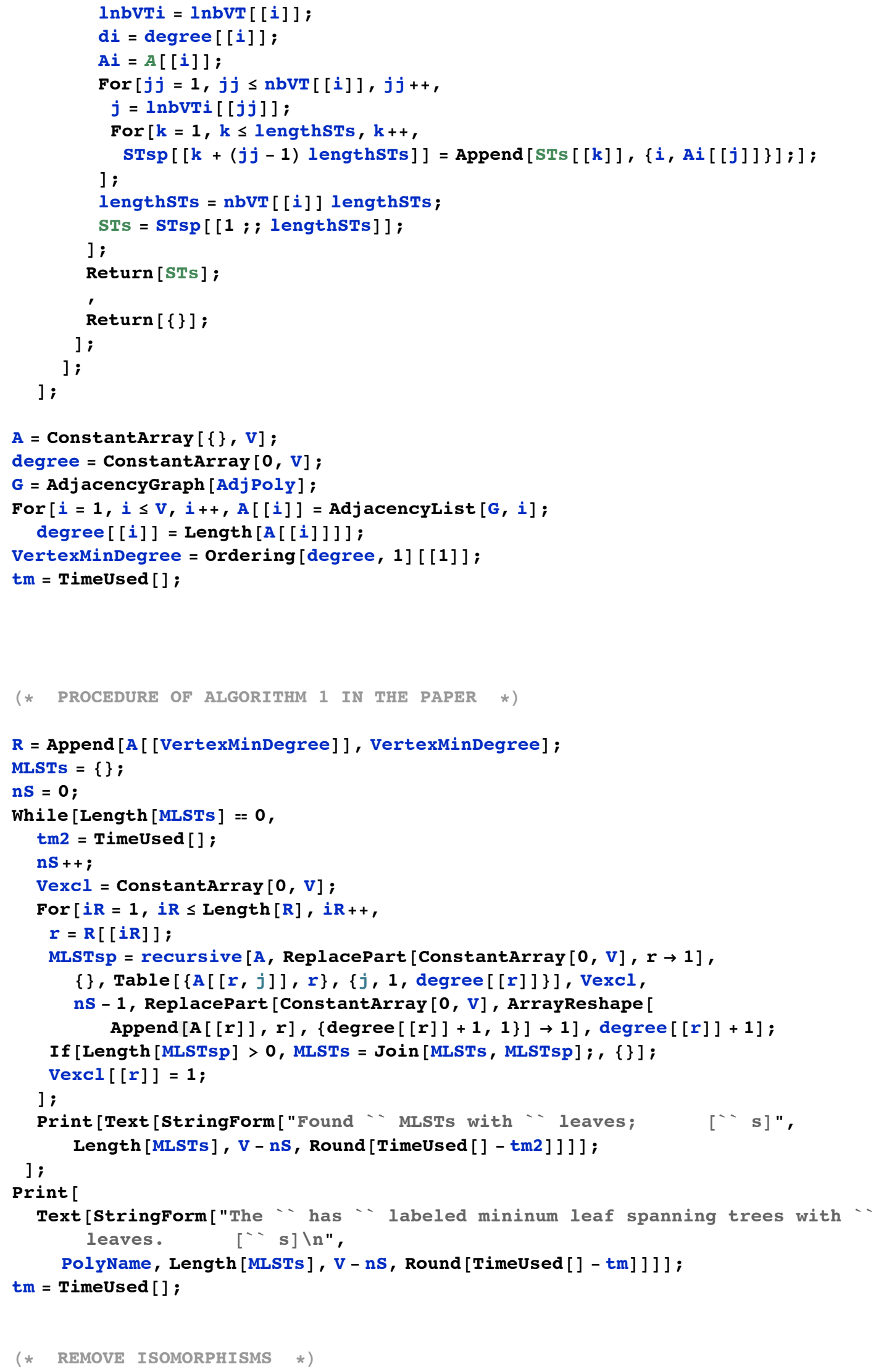




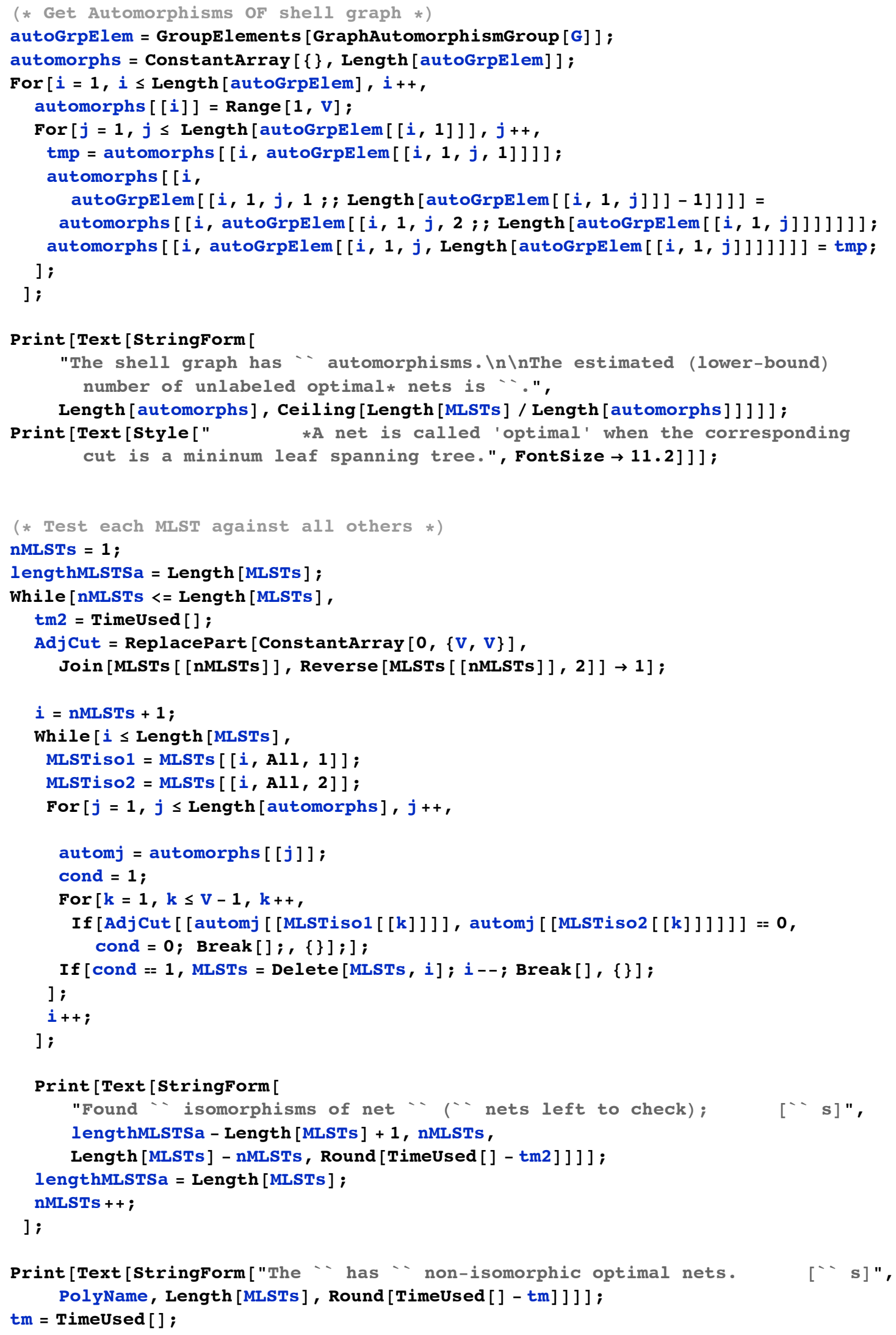




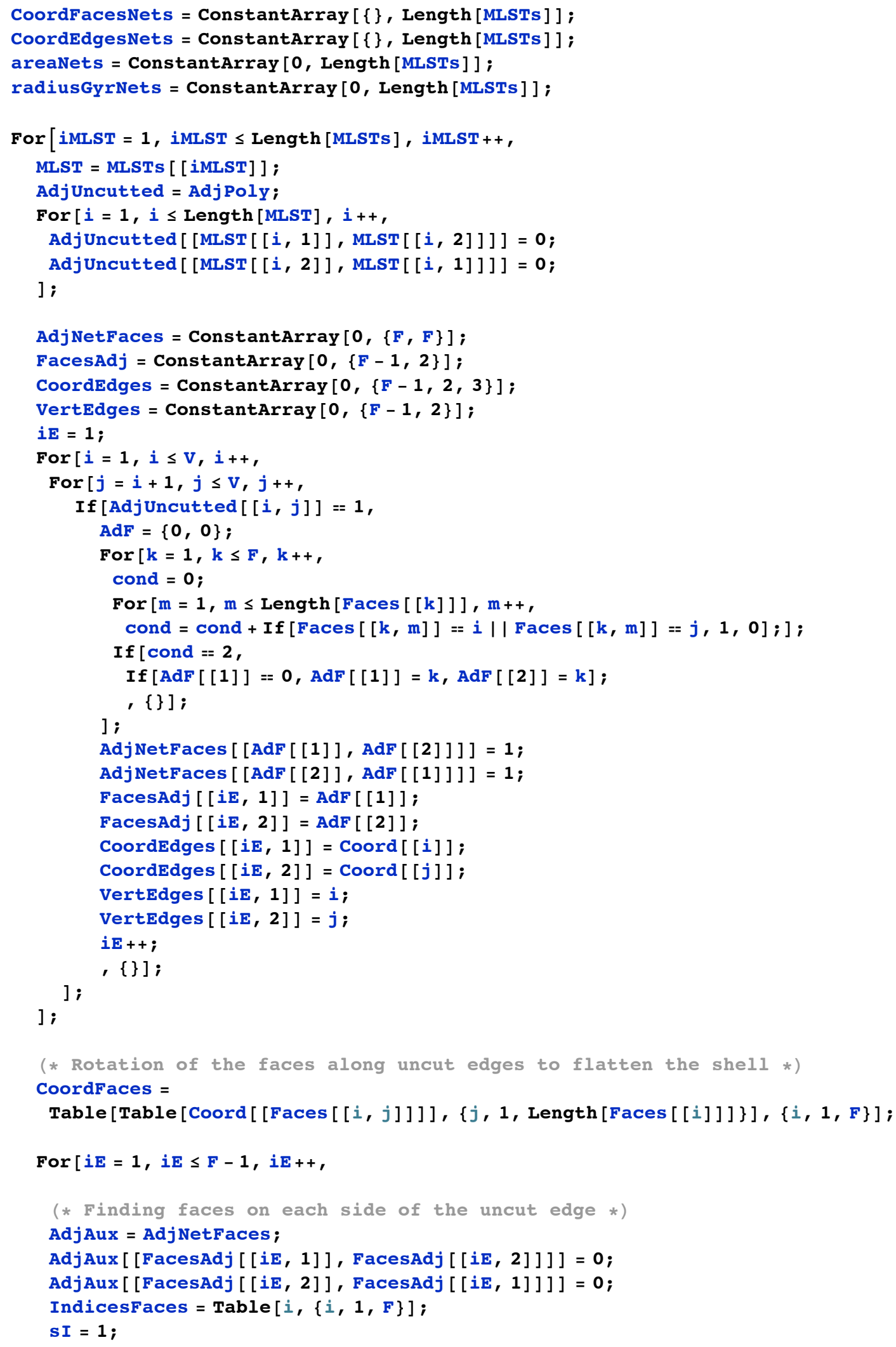




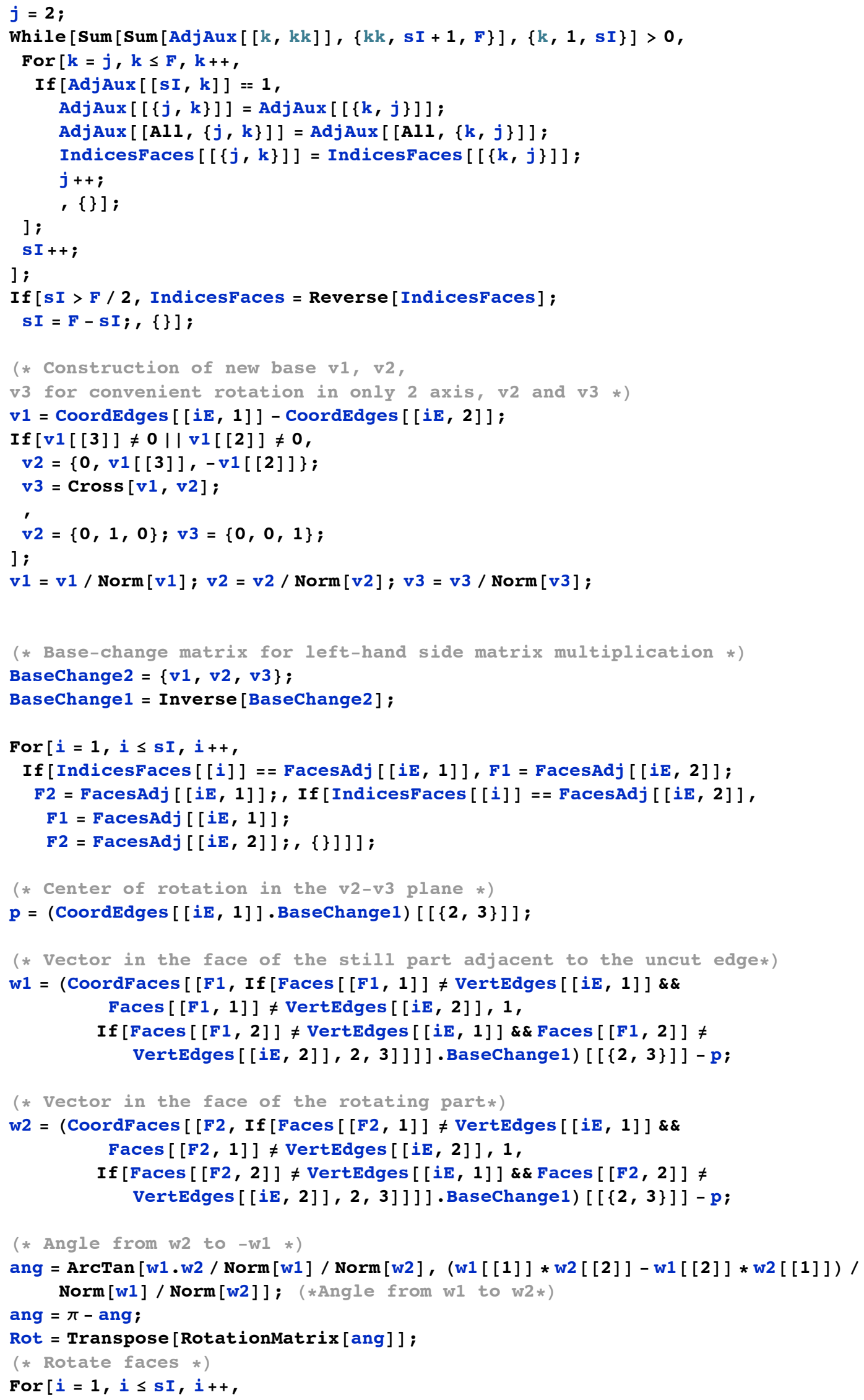




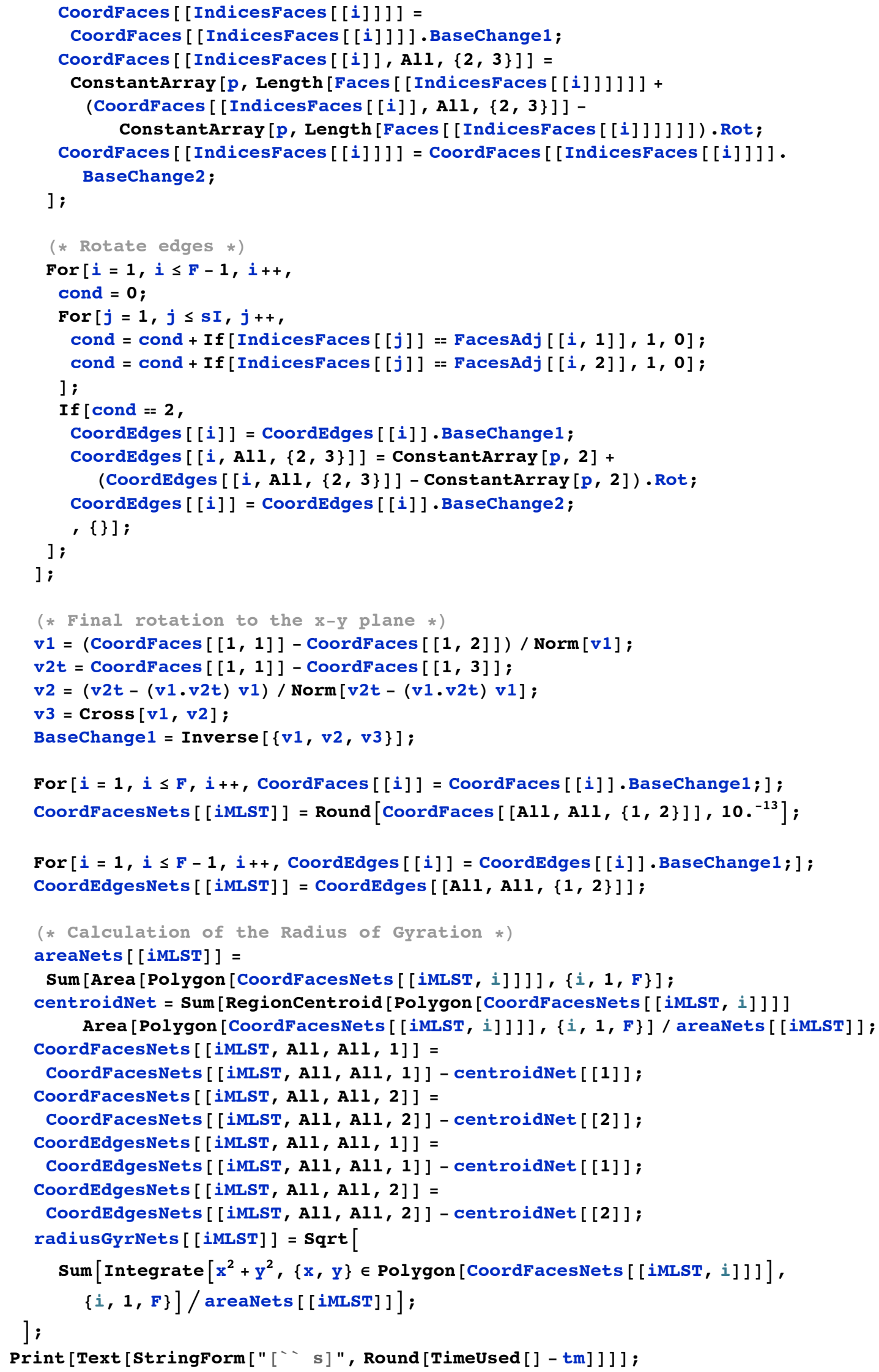




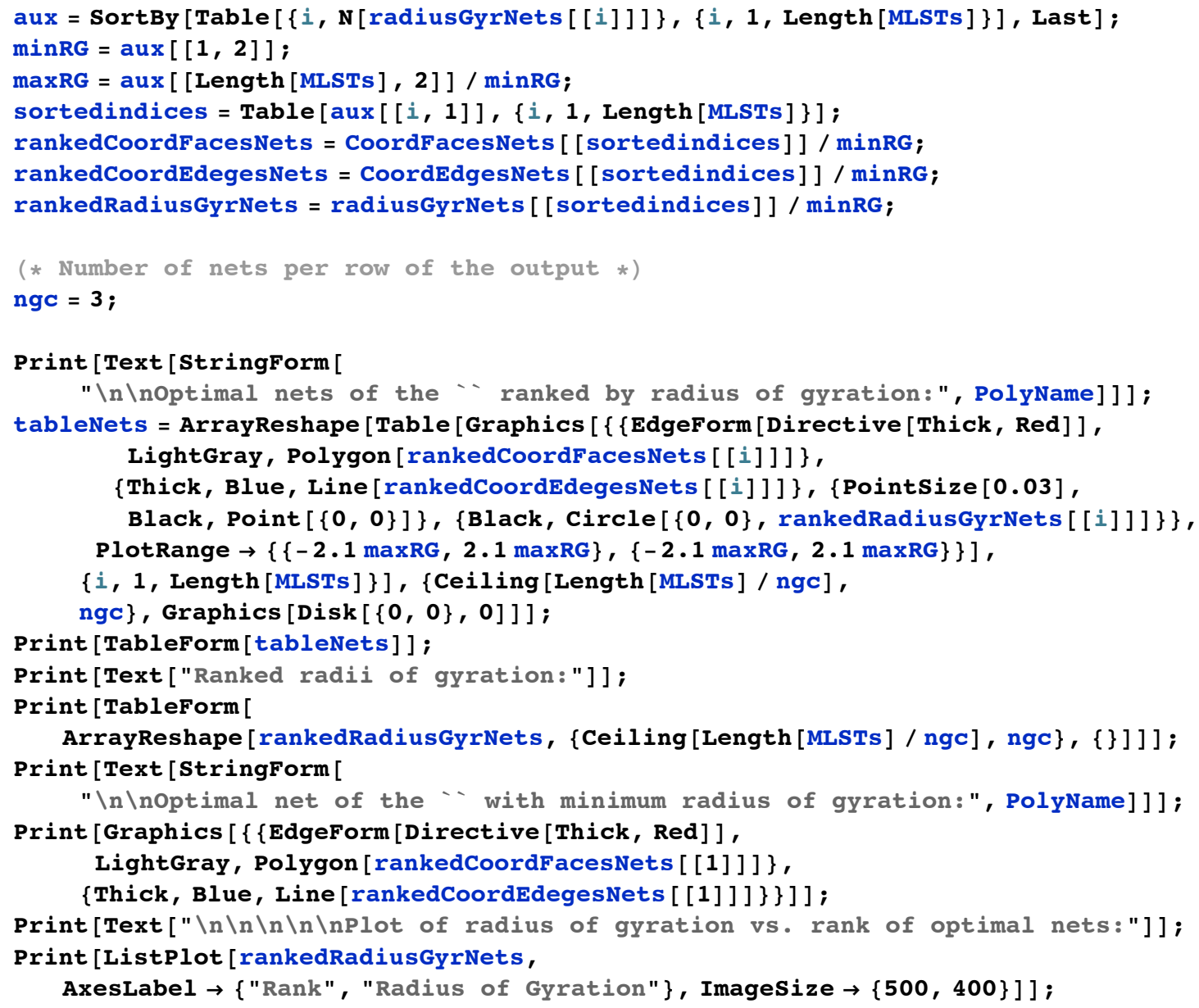




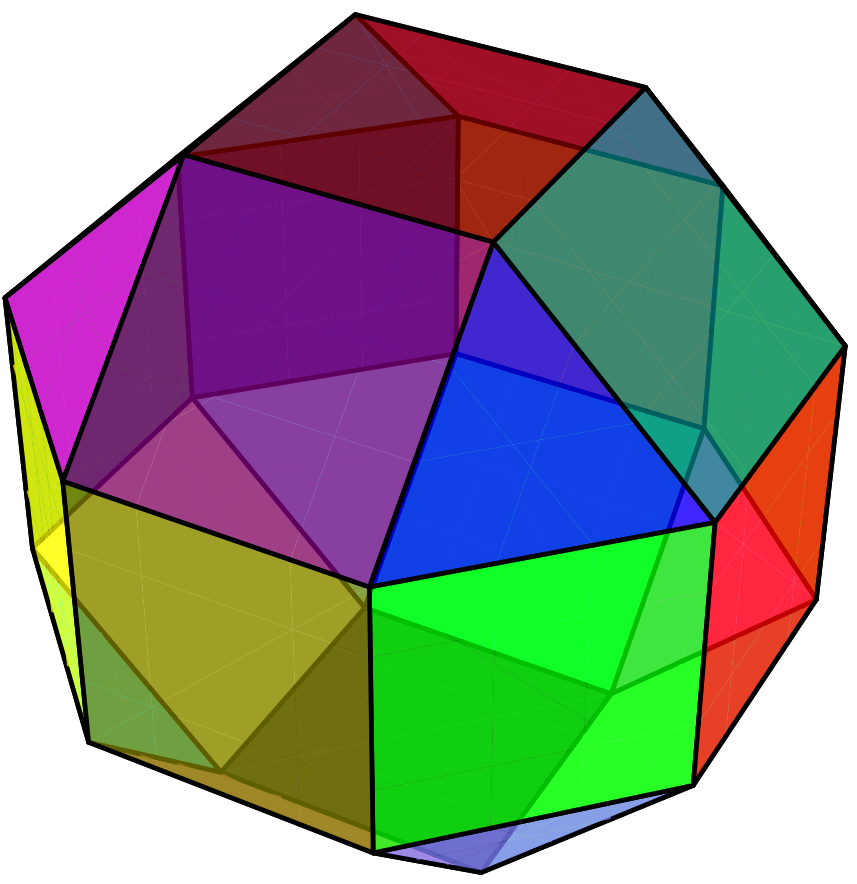

The SmallRhombicuboctahedron has 24 vertices, 26 faces, and 48 edges.

Found 0 MLSTs with 23 leaves; [0 s]

Found 0 MLSTs with 22 leaves; [0 s]

Found 0 MLSTs with 21 leaves; [0 s]

Found 0 MLSTs with 20 leaves; [0 s]

Found 0 MLSTs with 19 leaves; [0 s]

Found 0 MLSTs with 18 leaves; [0 s]

Found 0 MLSTs with 17 leaves; [2 s]

Found 0 MLSTs with 16 leaves; [8 s]

Found 1536 MLSTs with 15 leaves; [33 s]

The SmallRhombicuboctahedron has 1536 labeled mininum leaf spanning trees with 15 leaves. [43 s]

The shell graph has 48 automorphisms.

The estimated (lower-bound) number of unlabeled optimal $*$ nets is 32 .

*A net is called 'optimal' when the corresponding cut is a mininum leaf spanning tree.

Found 48 isomorphisms of net 1 (1488 nets left to check); [1 s]

Found 48 isomorphisms of net 2 (1440 nets left to check); [1 s]

Found 48 isomorphisms of net 3 (1392 nets left to check); [1 s]

Found 48 isomorphisms of net 4 (1344 nets left to check); [1 s]

Found 48 isomorphisms of net 5 (1296 nets left to check); [1 s]

Found 48 isomorphisms of net 6 (1248 nets left to check); [1 s] 
Found 48 isomorphisms of net 7 (1200 nets left to check); [1 s]

Found 48 isomorphisms of net 8 (1152 nets left to check); [1 s]

Found 48 isomorphisms of net 9 (1104 nets left to check); [1 s]

Found 48 isomorphisms of net 10 (1056 nets left to check); [1 s]

Found 48 isomorphisms of net 11 (1008 nets left to check); [1 s]

Found 48 isomorphisms of net 12 (960 nets left to check); [1 s]

Found 48 isomorphisms of net 13 (912 nets left to check); [1 s]

Found 48 isomorphisms of net 14 (864 nets left to check); [1 s]

Found 48 isomorphisms of net 15 (816 nets left to check); [1 s]

Found 48 isomorphisms of net 16 (768 nets left to check); [1 s]

Found 48 isomorphisms of net 17 (720 nets left to check); [1 s]

Found 48 isomorphisms of net 18 (672 nets left to check); [1 s]

Found 48 isomorphisms of net 19 (624 nets left to check); [0 s]

Found 48 isomorphisms of net 20 (576 nets left to check); [0 s]

Found 48 isomorphisms of net 21 (528 nets left to check); [0 s]

Found 48 isomorphisms of net 22 (480 nets left to check); [0 s]

Found 48 isomorphisms of net 23 (432 nets left to check); [0 s]

Found 48 isomorphisms of net 24 (384 nets left to check); [0 s]

Found 48 isomorphisms of net 25 (336 nets left to check); [0 s]

Found 48 isomorphisms of net 26 (288 nets left to check); [0 s]

Found 48 isomorphisms of net 27 (240 nets left to check); [0 s]

Found 48 isomorphisms of net 28 (192 nets left to check); [0 s]

Found 48 isomorphisms of net 29 (144 nets left to check); [0 s]

Found 48 isomorphisms of net 30 (96 nets left to check); [0 s]

Found 48 isomorphisms of net 31 (48 nets left to check); [0 s]

Found 48 isomorphisms of net 32 (0 nets left to check); [0 s]

The SmallRhombicuboctahedron has 32 non-isomorphic optimal nets. [18 s]

$[15 \mathrm{~s}]$

Optimal nets of the SmallRhombicuboctahedron ranked by radius of gyration:
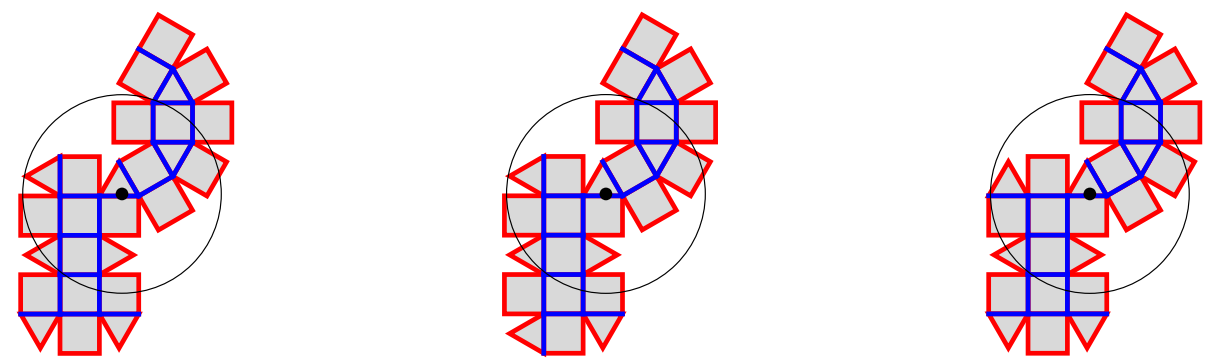

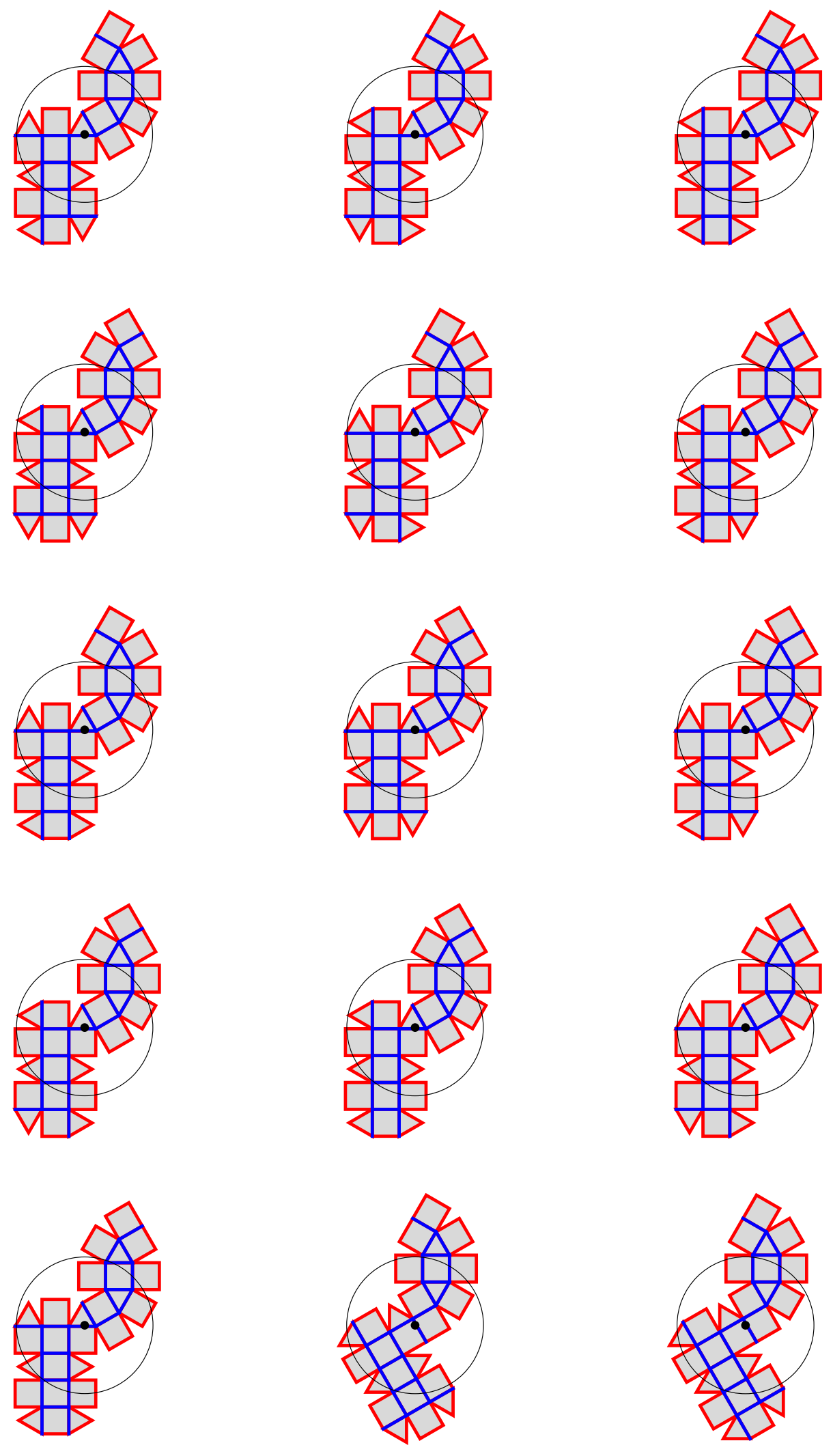

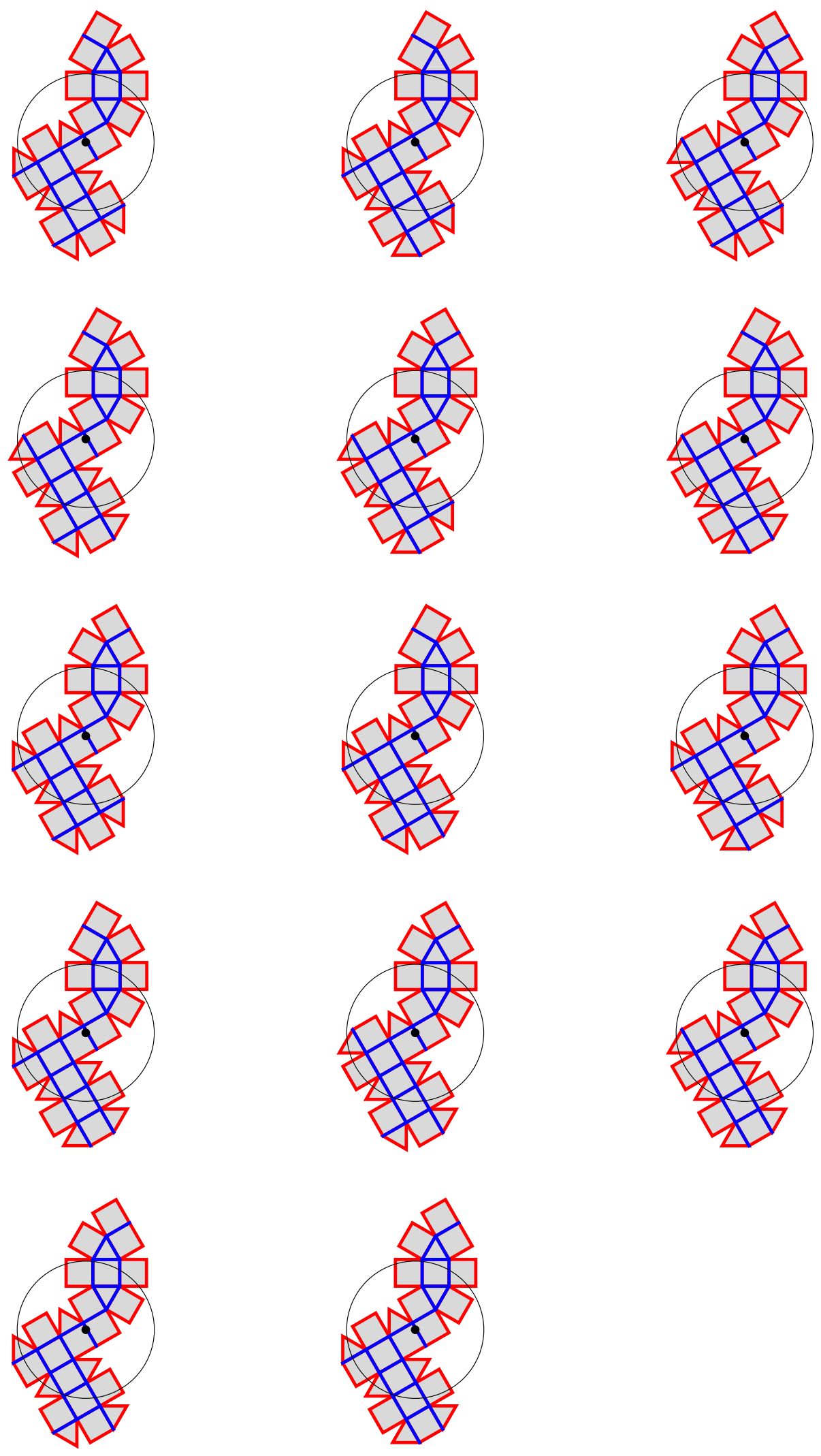

Ranked radii of gyration: 


$\begin{array}{lll}1 . & 1.00099 & 1.00109 \\ 1.00207 & 1.00242 & 1.00341 \\ 1.00343 & 1.0035 & 1.0044 \\ 1.00448 & 1.00453 & 1.0055 \\ 1.00586 & 1.00683 & 1.00694 \\ 1.00791 & 1.00913 & 1.00966 \\ 1.01026 & 1.01078 & 1.01118 \\ 1.01158 & 1.01169 & 1.0121 \\ 1.0123 & 1.0127 & 1.01281 \\ 1.01322 & 1.01363 & 1.01413 \\ 1.01475 & 1.01525 & 0\end{array}$

Optimal net of the SmallRhombicuboctahedron with minimum radius of gyration:

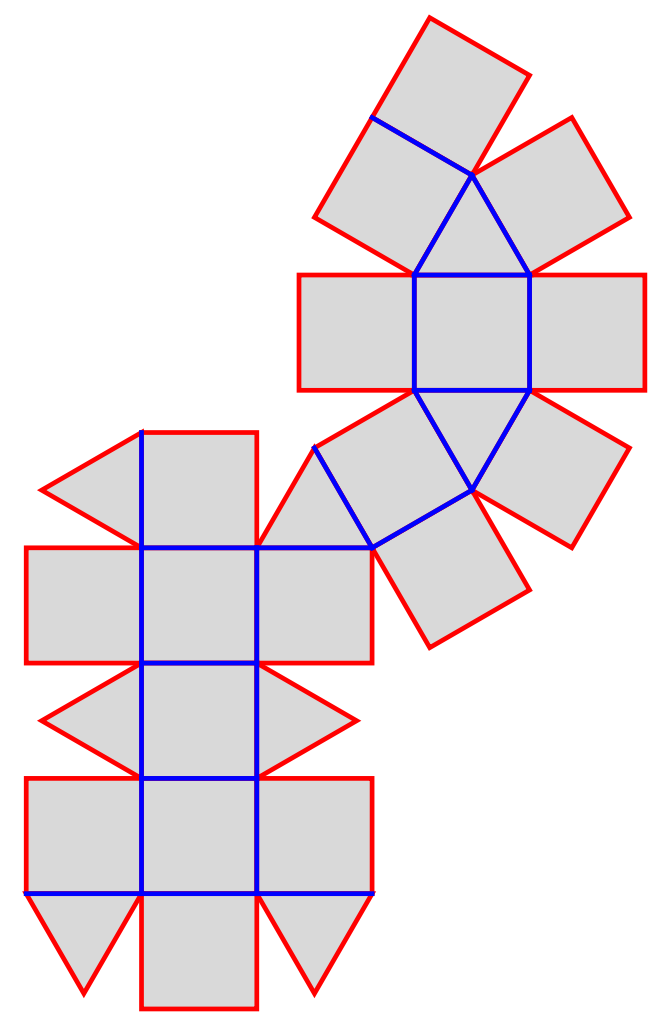

Plot of radius of gyration vs. rank of optimal nets: 


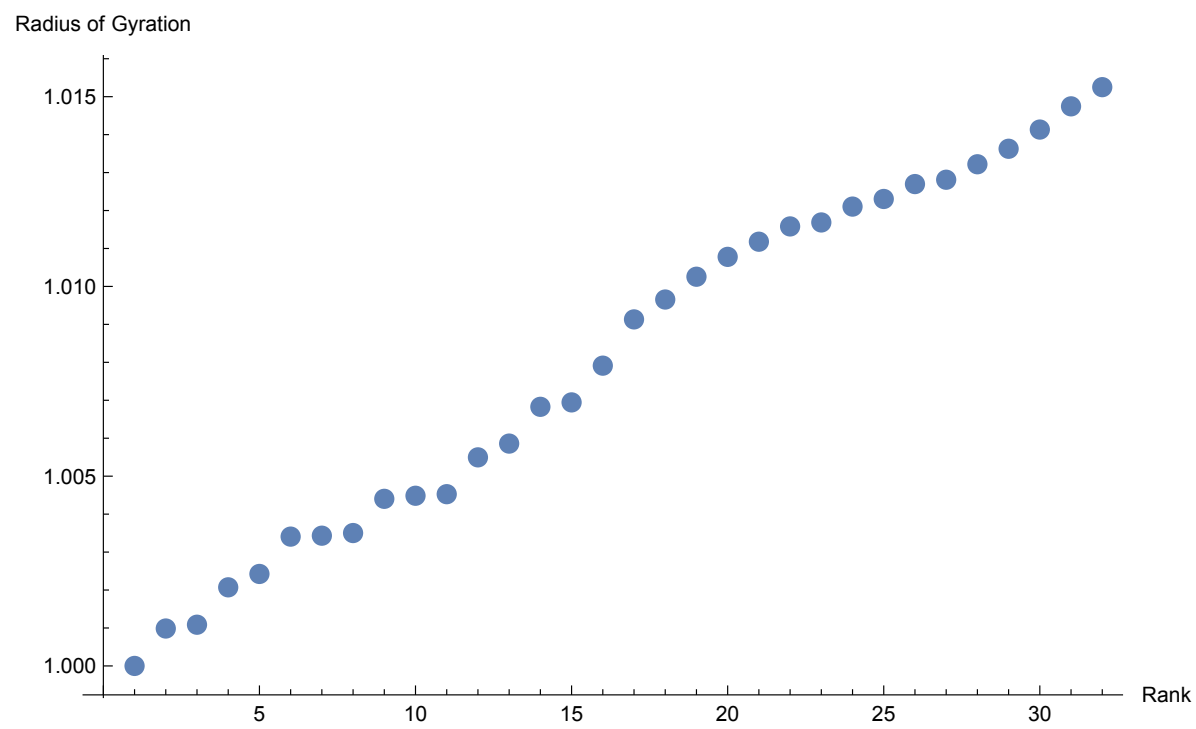




\section{S9. SOURCE CODE FOR OPEN SHELLS}

In this sections we print the source code of a Mathematica implementation of the algorithm presented in Sec. S3. This is the code that can be found in the file Procedure3_for_open_shells.nb available at URL https://journals.aps.org/prl/supplemental/ 10.1103/PhysRevLett.120.188001. This routine for shells with a hole needs the same inputs as the one for closed shells, and, additionally, the list of the vertices adjacent to the open face(s). During its execution the script outputs informations about the progress of the algorithm, and returns a list of all the nets with the maximum number of vertex connections. This list is sorted by the radius of gyration. The script also produces a plot of the ranked radii of gyration for the optimal nets found by the algorithm. The particular open shell treated by script printed here is the structure presented in Fig. 2e of the main paper, the Small Rhombicuboctahedron with the top nine faces removed.

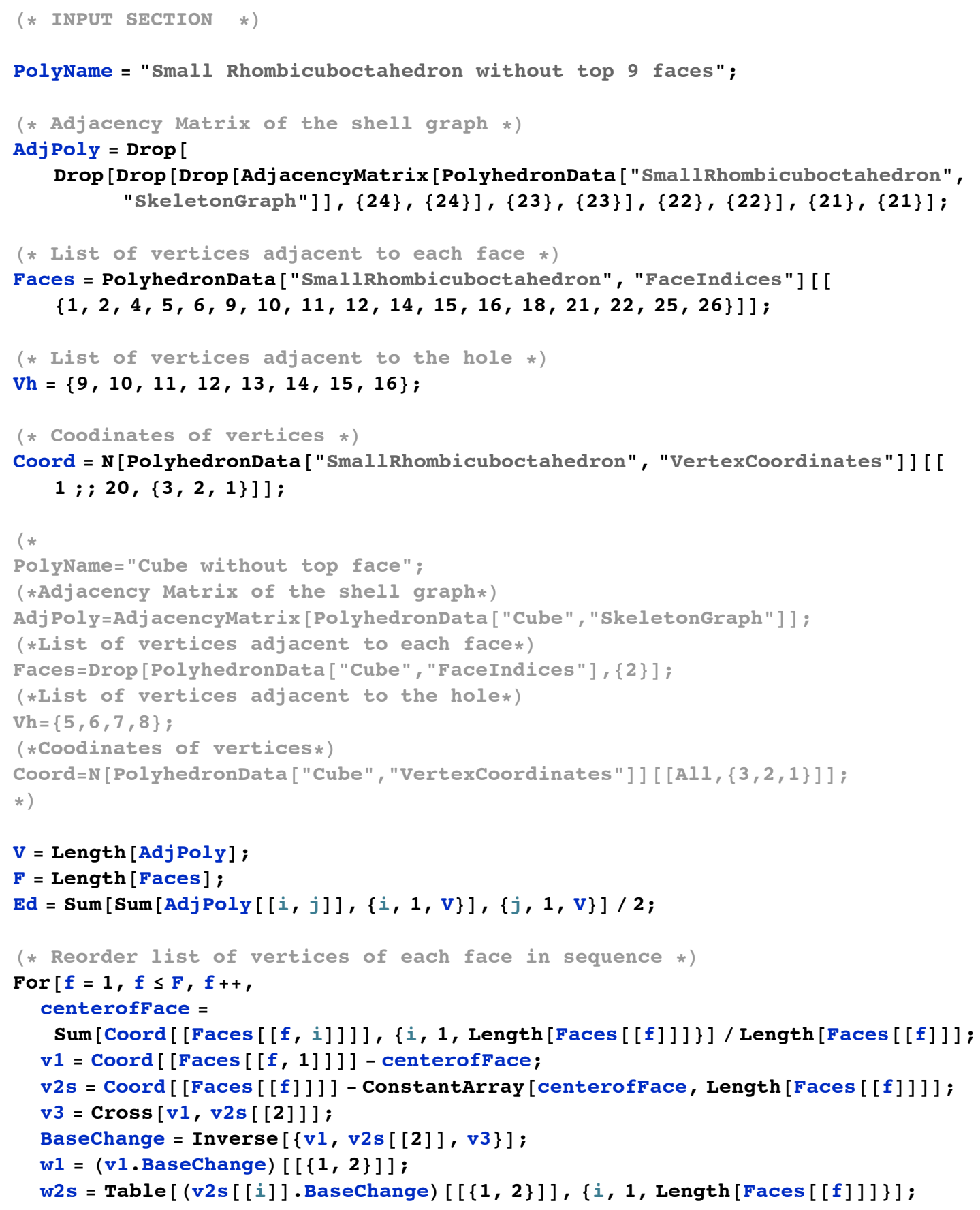




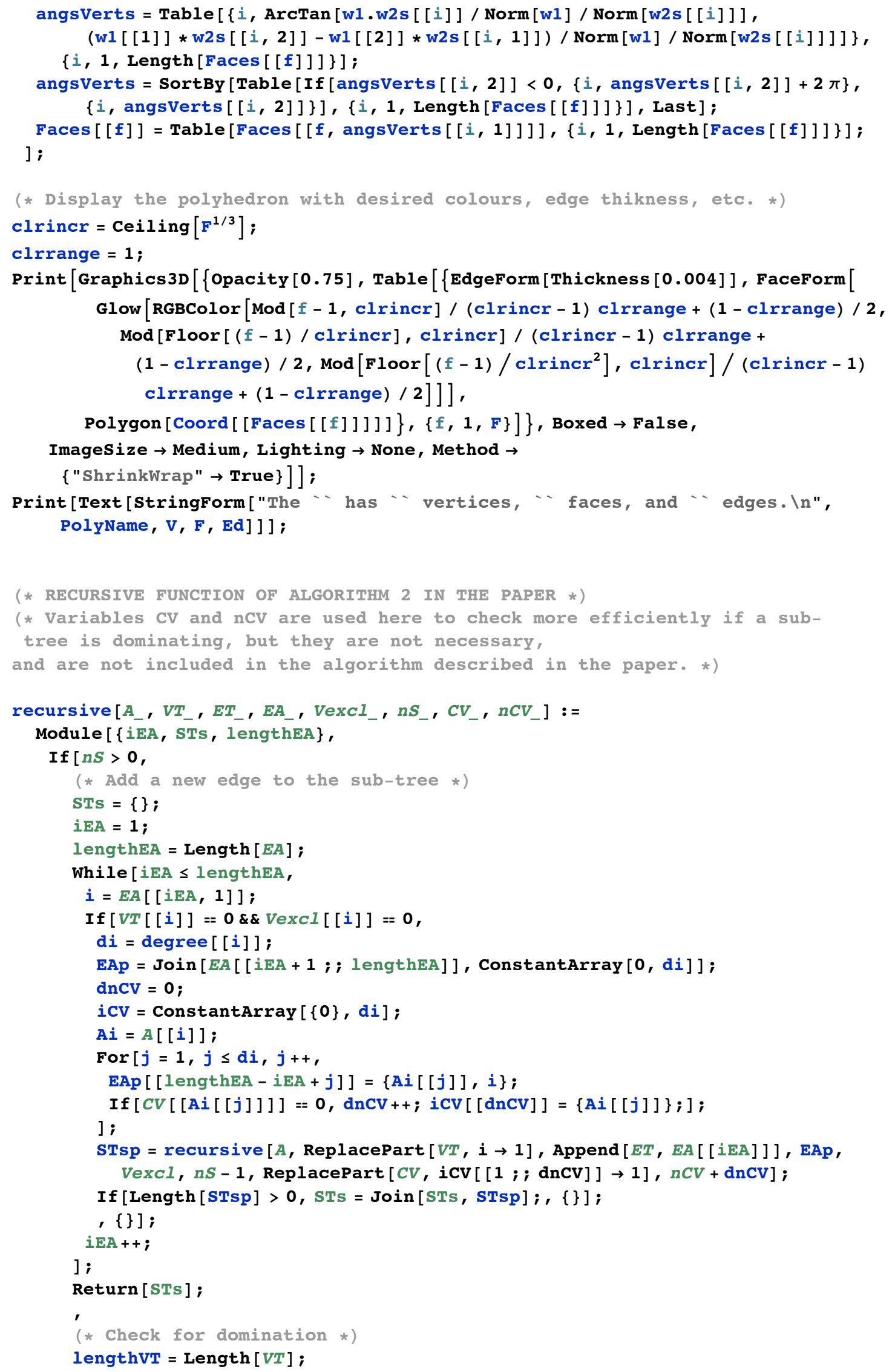




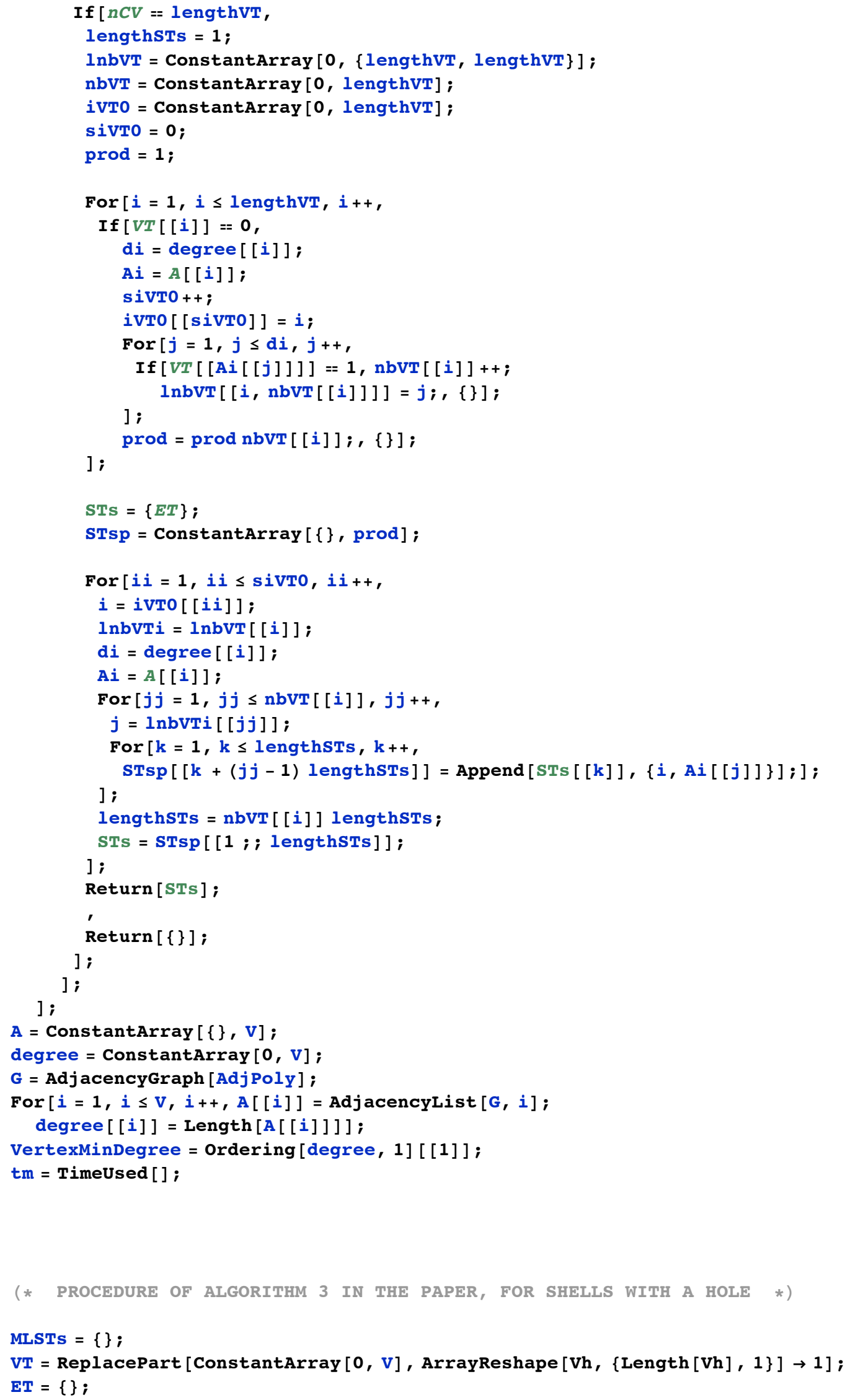




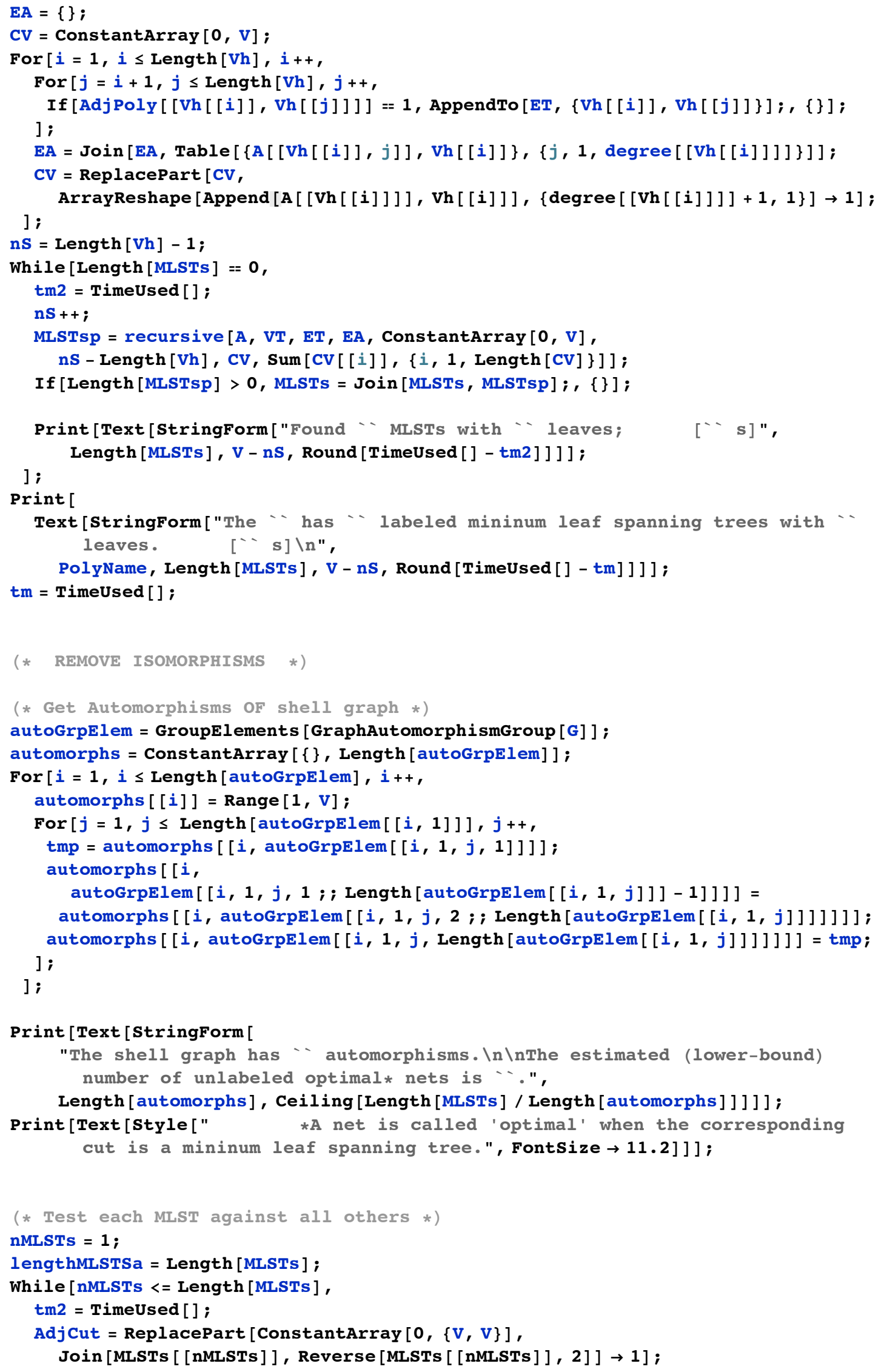




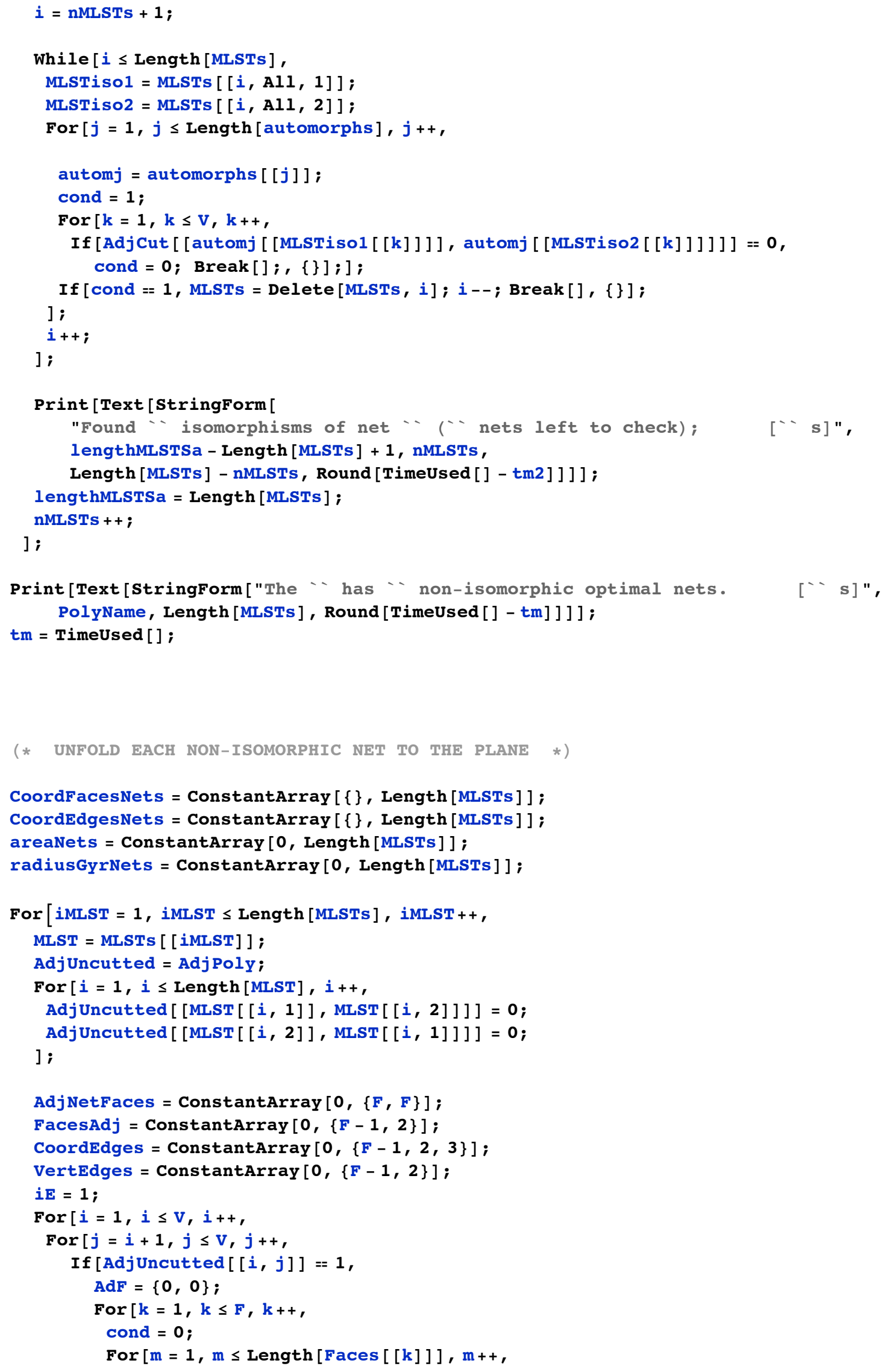




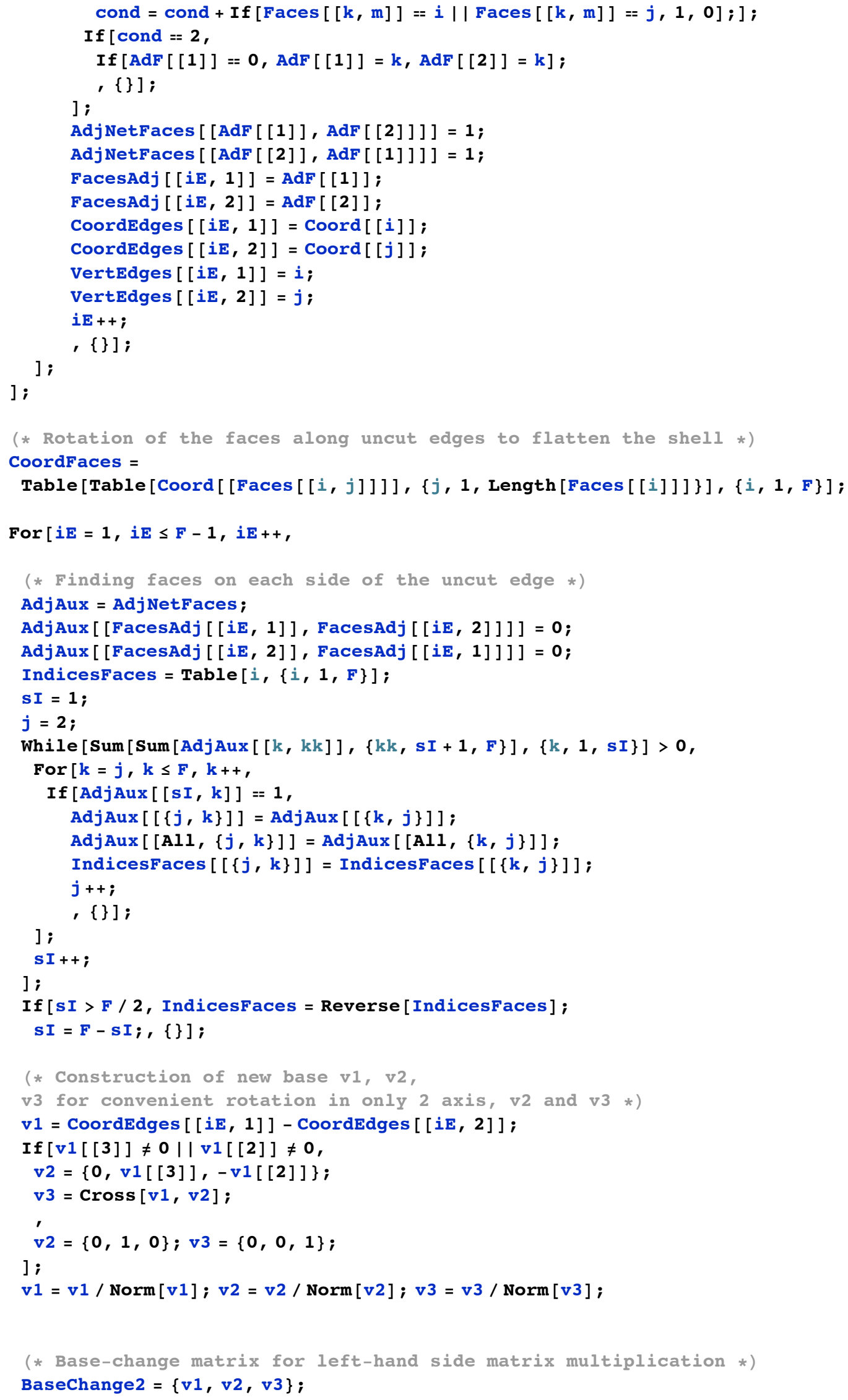




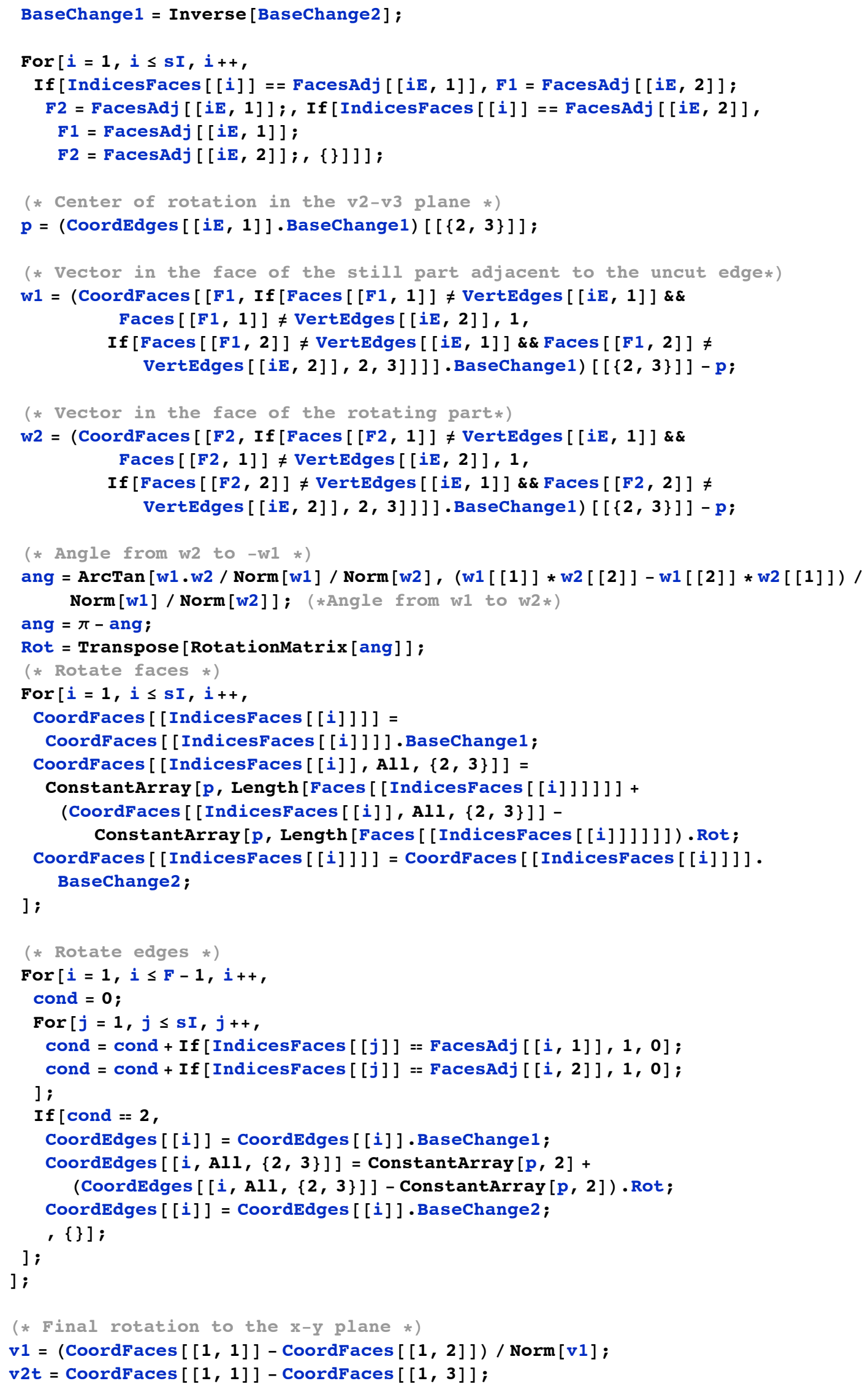




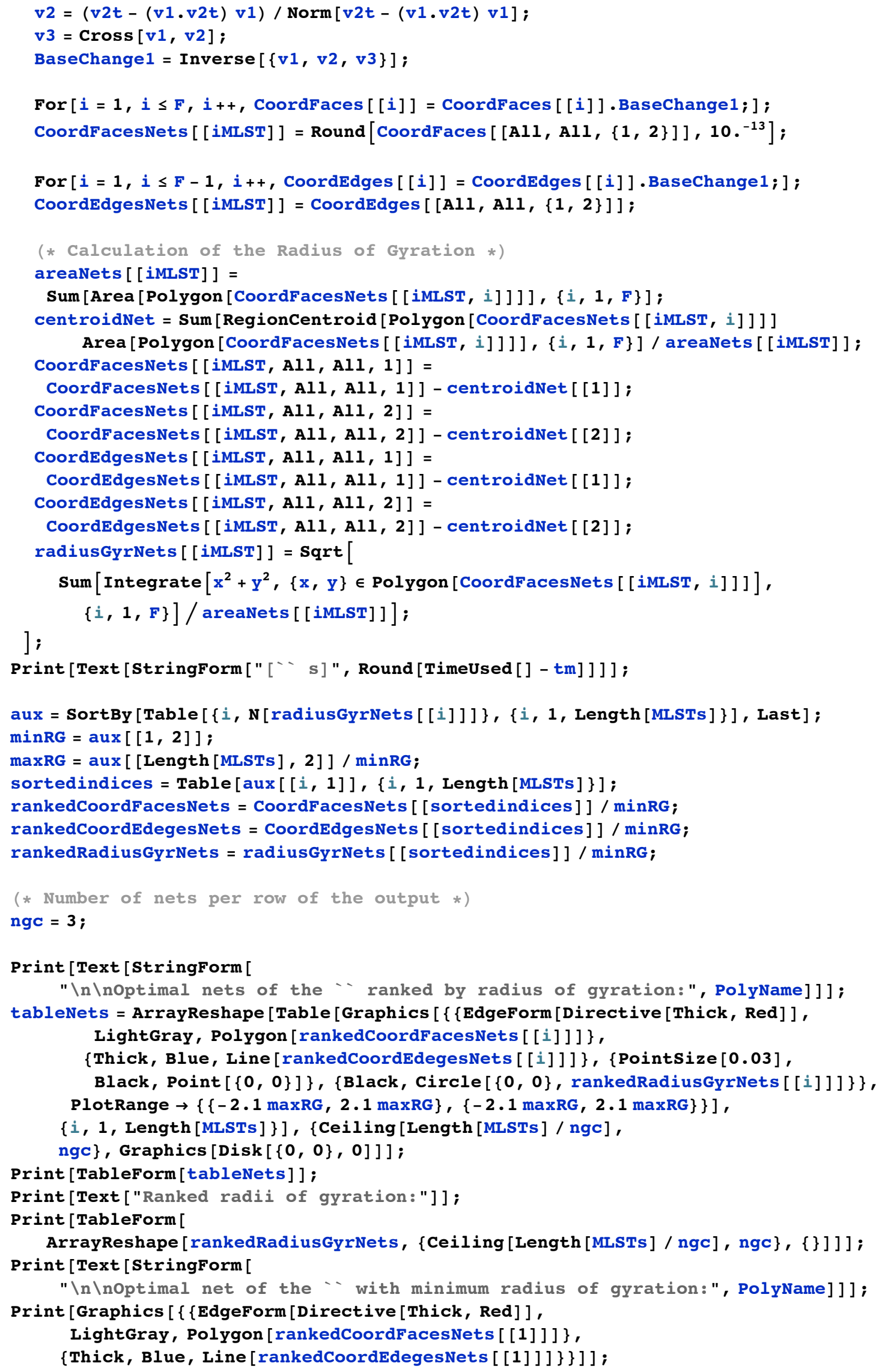


Print [Text [" $\backslash n \backslash n \backslash n \backslash n \backslash n P l o t$ of radius of gyration vs. rank of optimal nets:"]]; Print [ListPlot [rankedRadiusGyrNets, AxesLabel $\rightarrow\{$ "Rank", "Radius of Gyration" $\}$, ImageSize $\rightarrow\{500,400\}]]$;

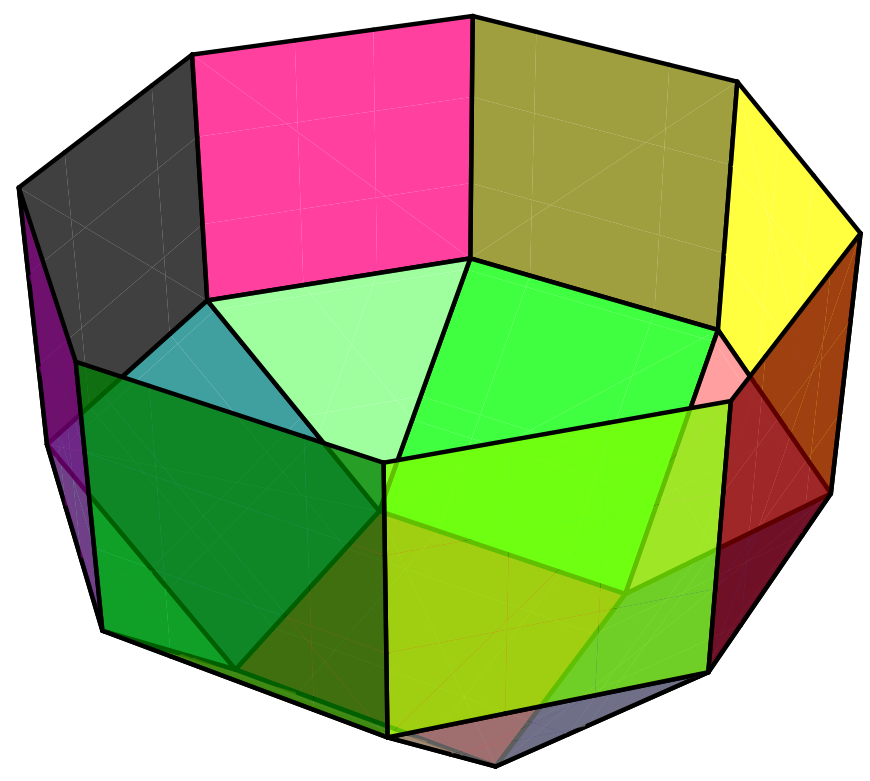

The Small Rhombicuboctahedron without top 9 faces has 20 vertices, 17 faces, and 36 edges.

Found 0 MLSTs with 12 leaves; [0 s]

Found 0 MLSTs with 11 leaves; [0 s]

Found 0 MLSTs with 10 leaves; [0 s]

Found 720 MLSTs with 9 leaves; [0 s]

The Small Rhombicuboctahedron without top 9 faces has 720 labeled mininum leaf spanning trees with 9 leaves.

The shell graph has 8 automorphisms.

The estimated (lower-bound) number of unlabeled optimal $*$ nets is 90 .

*A net is called 'optimal' when the corresponding cut is a mininum leaf spanning tree.

Found 8 isomorphisms of net 1 (712 nets left to check); [0 s]

Found 8 isomorphisms of net 2 (704 nets left to check); [0 s]

Found 8 isomorphisms of net 3 (696 nets left to check); [0 s]

Found 8 isomorphisms of net 4 (688 nets left to check); [0 s]

Found 8 isomorphisms of net 5 (680 nets left to check); [0 s]

Found 8 isomorphisms of net 6 (672 nets left to check); [0 s]

Found 8 isomorphisms of net 7 (664 nets left to check); [0 s]

Found 8 isomorphisms of net 8 (656 nets left to check); [0 s] 
Found 8 isomorphisms of net 9 (648 nets left to check); [0 s]

Found 8 isomorphisms of net 10 (640 nets left to check); [0 s]

Found 8 isomorphisms of net 11 (632 nets left to check); [0 s]

Found 8 isomorphisms of net 12 (624 nets left to check); [0 s]

Found 8 isomorphisms of net 13 (616 nets left to check); [0 s]

Found 8 isomorphisms of net 14 (608 nets left to check); [0 s]

Found 8 isomorphisms of net 15 (600 nets left to check); [0 s]

Found 8 isomorphisms of net 16 (592 nets left to check); [0 s]

Found 8 isomorphisms of net 17 (584 nets left to check); [0 s]

Found 8 isomorphisms of net 18 (576 nets left to check); [0 s]

Found 8 isomorphisms of net 19 (568 nets left to check); [0 s]

Found 8 isomorphisms of net 20 (560 nets left to check); [0 s]

Found 8 isomorphisms of net 21 (552 nets left to check); [0 s]

Found 8 isomorphisms of net 22 (544 nets left to check); [0 s]

Found 8 isomorphisms of net 23 (536 nets left to check); [0 s]

Found 8 isomorphisms of net 24 (528 nets left to check); [0 s]

Found 8 isomorphisms of net 25 (520 nets left to check); [0 s]

Found 8 isomorphisms of net 26 (512 nets left to check); [0 s]

Found 8 isomorphisms of net 27 (504 nets left to check); [0 s]

Found 8 isomorphisms of net 28 (496 nets left to check); [0 s]

Found 8 isomorphisms of net 29 (488 nets left to check); [0 s]

Found 8 isomorphisms of net 30 (480 nets left to check); [0 s]

Found 8 isomorphisms of net 31 (472 nets left to check); [0 s]

Found 8 isomorphisms of net 32 (464 nets left to check); [0 s]

Found 8 isomorphisms of net 33 (456 nets left to check); [0 s]

Found 8 isomorphisms of net 34 (448 nets left to check); [0 s]

Found 8 isomorphisms of net 35 (440 nets left to check); [0 s]

Found 8 isomorphisms of net 36 (432 nets left to check); [0 s]

Found 8 isomorphisms of net 37 (424 nets left to check); [0 s]

Found 8 isomorphisms of net 38 (416 nets left to check); [0 s]

Found 8 isomorphisms of net 39 (408 nets left to check); [0 s]

Found 8 isomorphisms of net 40 (400 nets left to check); [0 s]

Found 8 isomorphisms of net 41 (392 nets left to check); [0 s]

Found 8 isomorphisms of net 42 (384 nets left to check); [0 s]

Found 8 isomorphisms of net 43 (376 nets left to check); [0 s]

Found 8 isomorphisms of net 44 (368 nets left to check); [0 s]

Found 8 isomorphisms of net 45 (360 nets left to check); [0 s]

Found 8 isomorphisms of net 46 (352 nets left to check); [0 s]

Found 8 isomorphisms of net 47 (344 nets left to check); [0 s] 
Found 8 isomorphisms of net 48 (336 nets left to check); [0 s]

Found 8 isomorphisms of net 49 (328 nets left to check); [0 s]

Found 8 isomorphisms of net 50 (320 nets left to check); [0 s]

Found 8 isomorphisms of net 51 (312 nets left to check); [0 s]

Found 8 isomorphisms of net 52 (304 nets left to check); [0 s]

Found 8 isomorphisms of net 53 (296 nets left to check); [0 s]

Found 8 isomorphisms of net 54 (288 nets left to check); [0 s]

Found 8 isomorphisms of net 55 ( 280 nets left to check); [0 s]

Found 8 isomorphisms of net 56 (272 nets left to check); [0 s]

Found 8 isomorphisms of net 57 (264 nets left to check); [0 s]

Found 8 isomorphisms of net 58 ( 256 nets left to check); [0 s]

Found 8 isomorphisms of net 59 ( 248 nets left to check); [0 s]

Found 8 isomorphisms of net 60 (240 nets left to check); [0 s]

Found 8 isomorphisms of net 61 (232 nets left to check); [0 s]

Found 8 isomorphisms of net 62 (224 nets left to check); [0 s]

Found 8 isomorphisms of net 63 (216 nets left to check); [0 s]

Found 8 isomorphisms of net 64 (208 nets left to check); [0 s]

Found 8 isomorphisms of net 65 (200 nets left to check); [0 s]

Found 8 isomorphisms of net 66 (192 nets left to check); [0 s]

Found 8 isomorphisms of net 67 (184 nets left to check); [0 s]

Found 8 isomorphisms of net 68 (176 nets left to check); [0 s]

Found 8 isomorphisms of net 69 (168 nets left to check); [0 s]

Found 8 isomorphisms of net 70 (160 nets left to check); [0 s]

Found 8 isomorphisms of net 71 (152 nets left to check); [0 s]

Found 8 isomorphisms of net 72 (144 nets left to check); [0 s]

Found 8 isomorphisms of net 73 (136 nets left to check); [0 s]

Found 8 isomorphisms of net 74 (128 nets left to check); [0 s]

Found 8 isomorphisms of net 75 (120 nets left to check); [0 s]

Found 8 isomorphisms of net 76 (112 nets left to check); [0 s]

Found 8 isomorphisms of net 77 (104 nets left to check); [0 s]

Found 8 isomorphisms of net 78 (96 nets left to check); [0 s]

Found 8 isomorphisms of net 79 ( 88 nets left to check); [0 s]

Found 8 isomorphisms of net 80 ( 80 nets left to check); [0 s]

Found 8 isomorphisms of net 81 (72 nets left to check); [0 s]

Found 8 isomorphisms of net 82 (64 nets left to check); [0 s]

Found 8 isomorphisms of net 83 (56 nets left to check); [0 s]

Found 8 isomorphisms of net 84 (48 nets left to check); [0 s]

Found 8 isomorphisms of net 85 (40 nets left to check); [0 s]

Found 8 isomorphisms of net 86 (32 nets left to check); [0 s] 
Found 8 isomorphisms of net 87 (24 nets left to check); [0 s]

Found 8 isomorphisms of net 88 (16 nets left to check); [0 s]

Found 8 isomorphisms of net 89 ( 8 nets left to check); [0 s]

Found 8 isomorphisms of net 90 (0 nets left to check); [0 s]

The Small Rhombicuboctahedron without top 9 faces has 90 non-isomorphic optimal nets. [13 s]

$[29 \mathrm{~s}]$

Optimal nets of the Small Rhombicuboctahedron without top 9 faces ranked by radius of gyration:
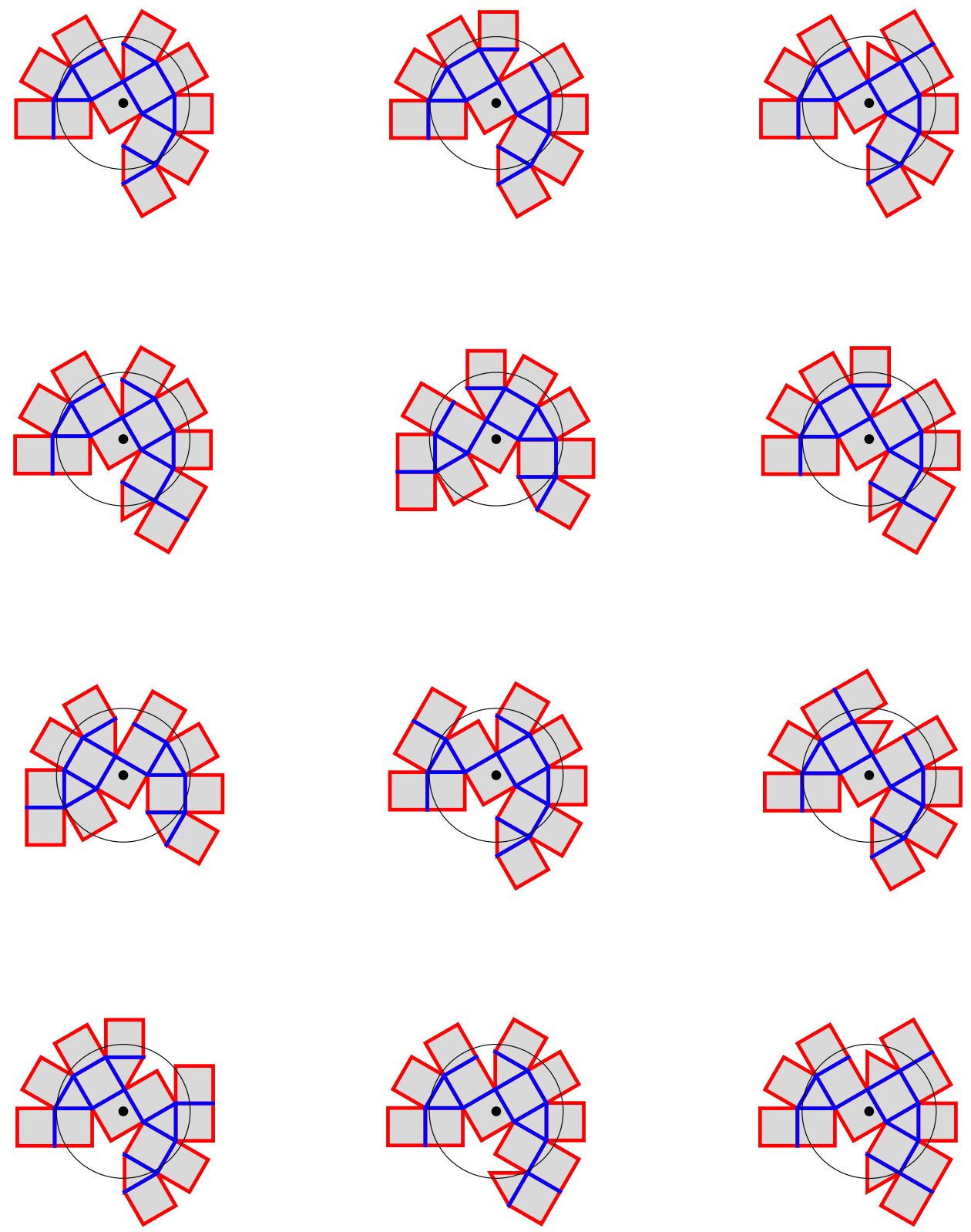

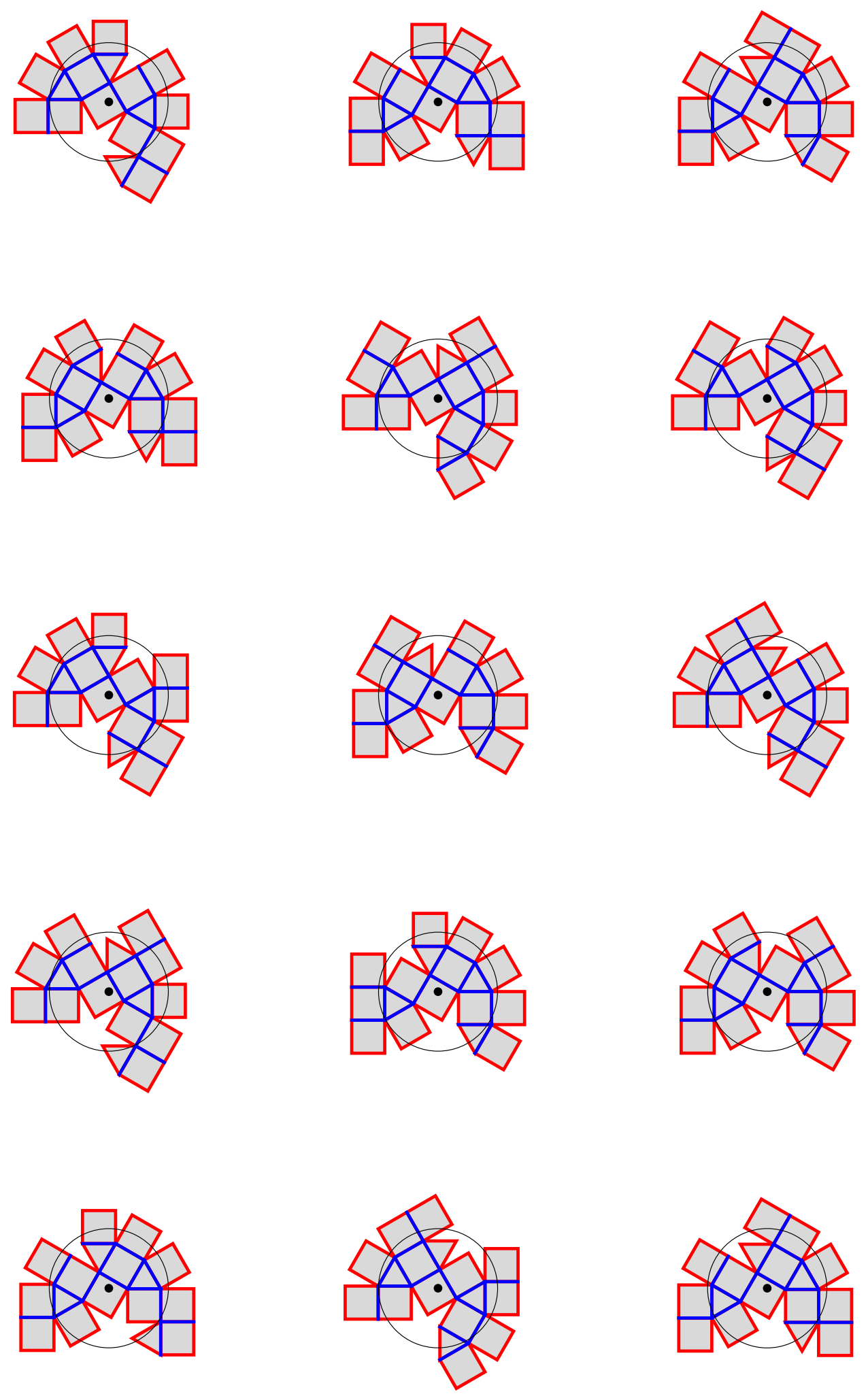

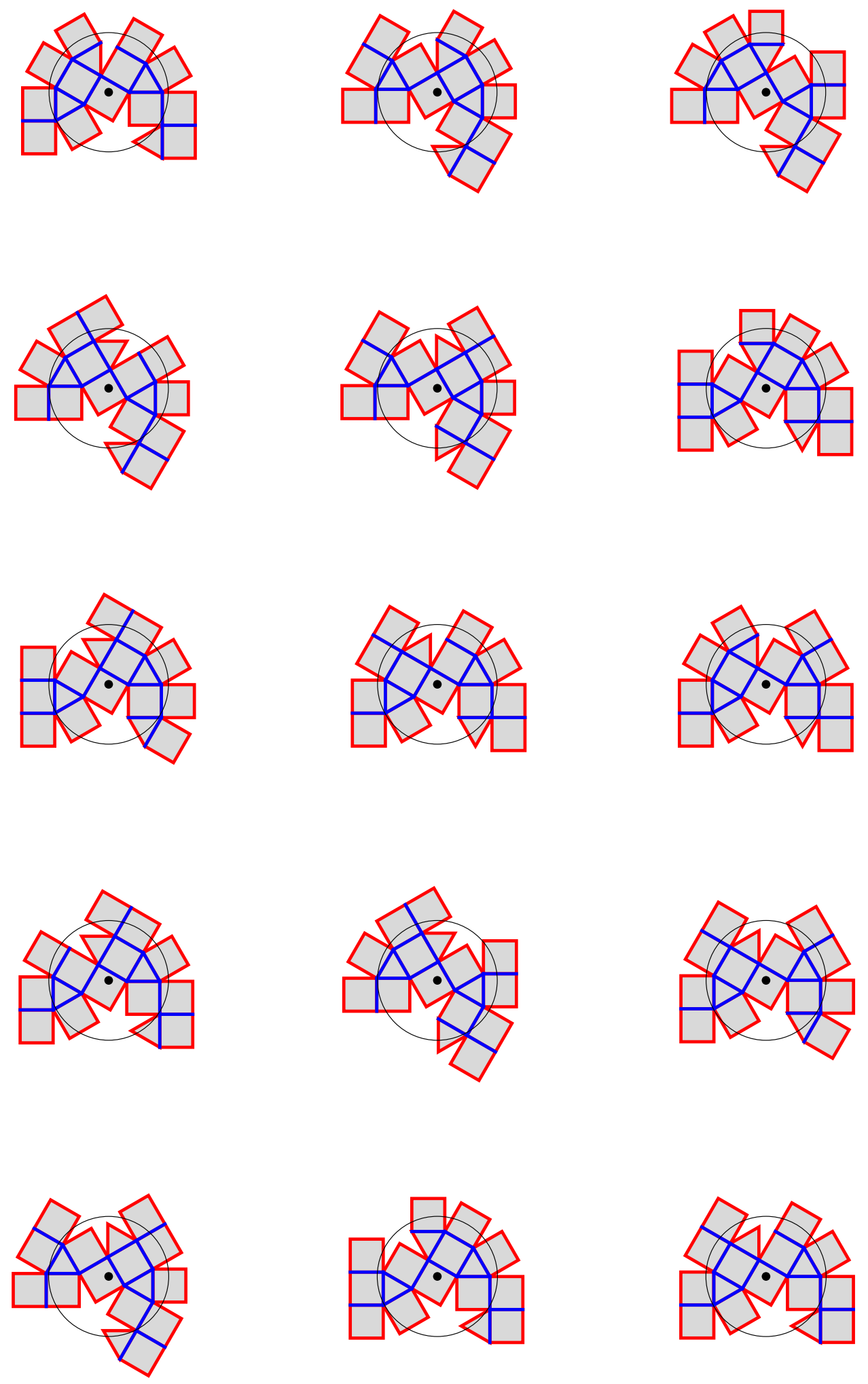

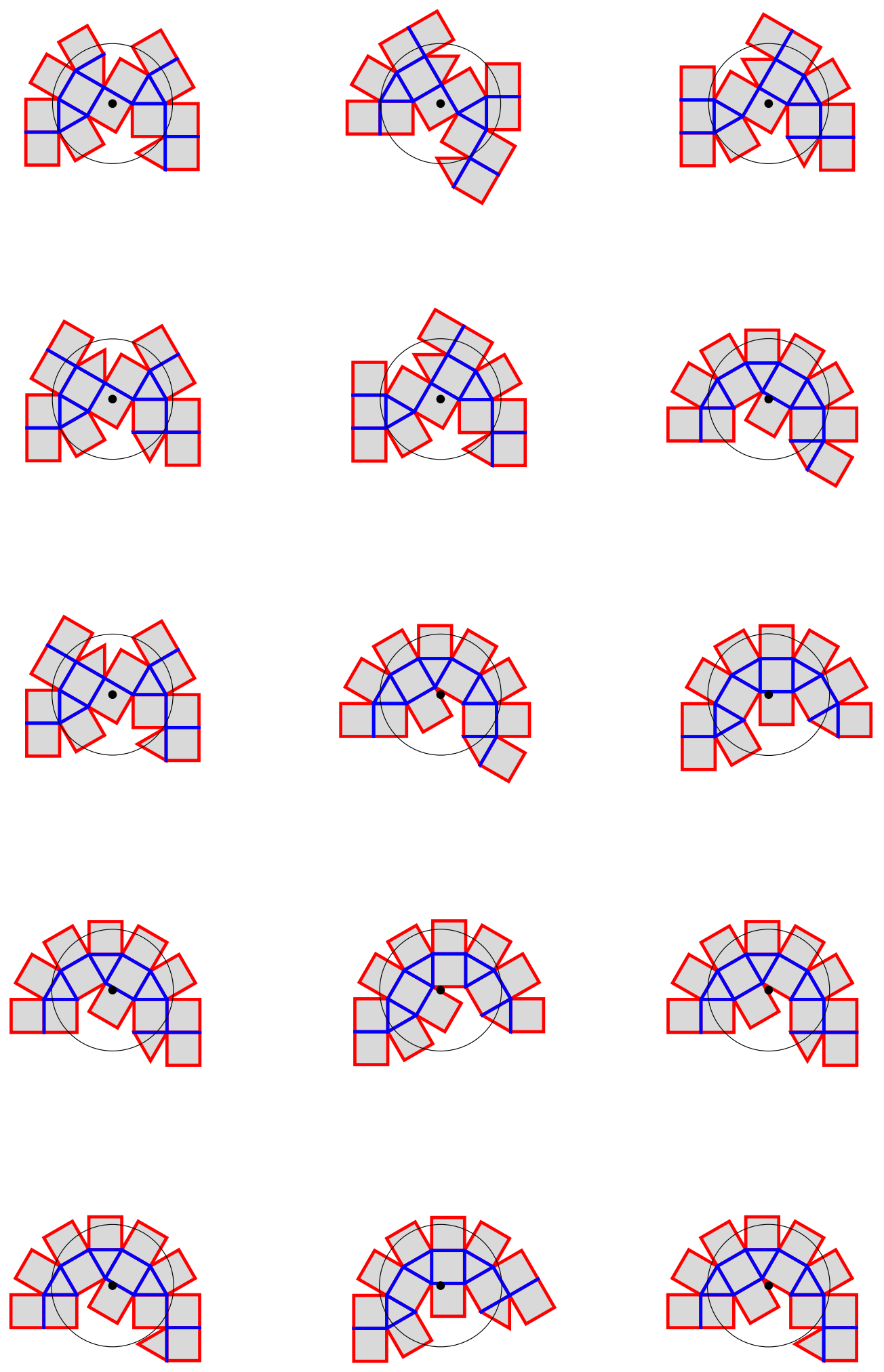
S38
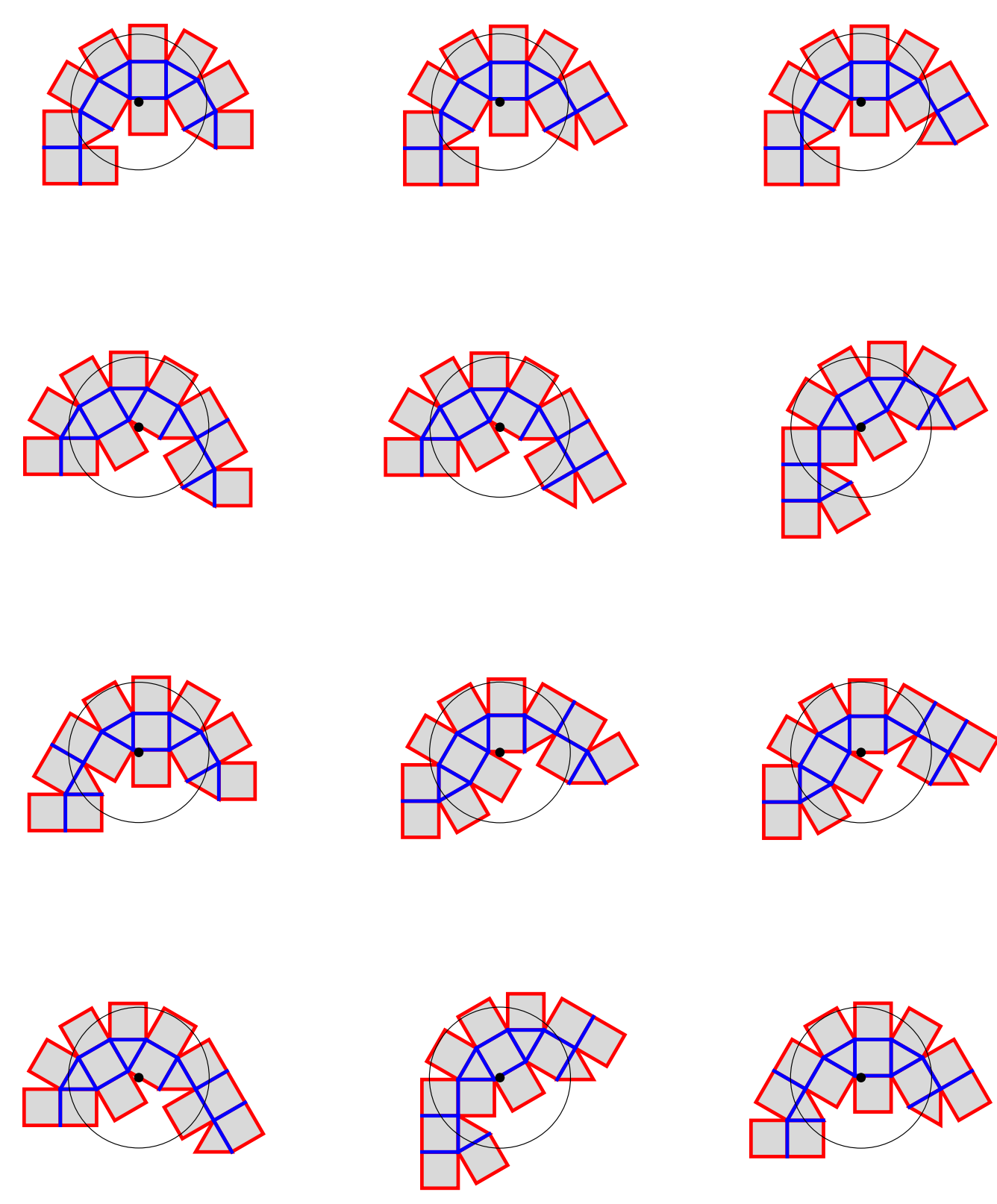

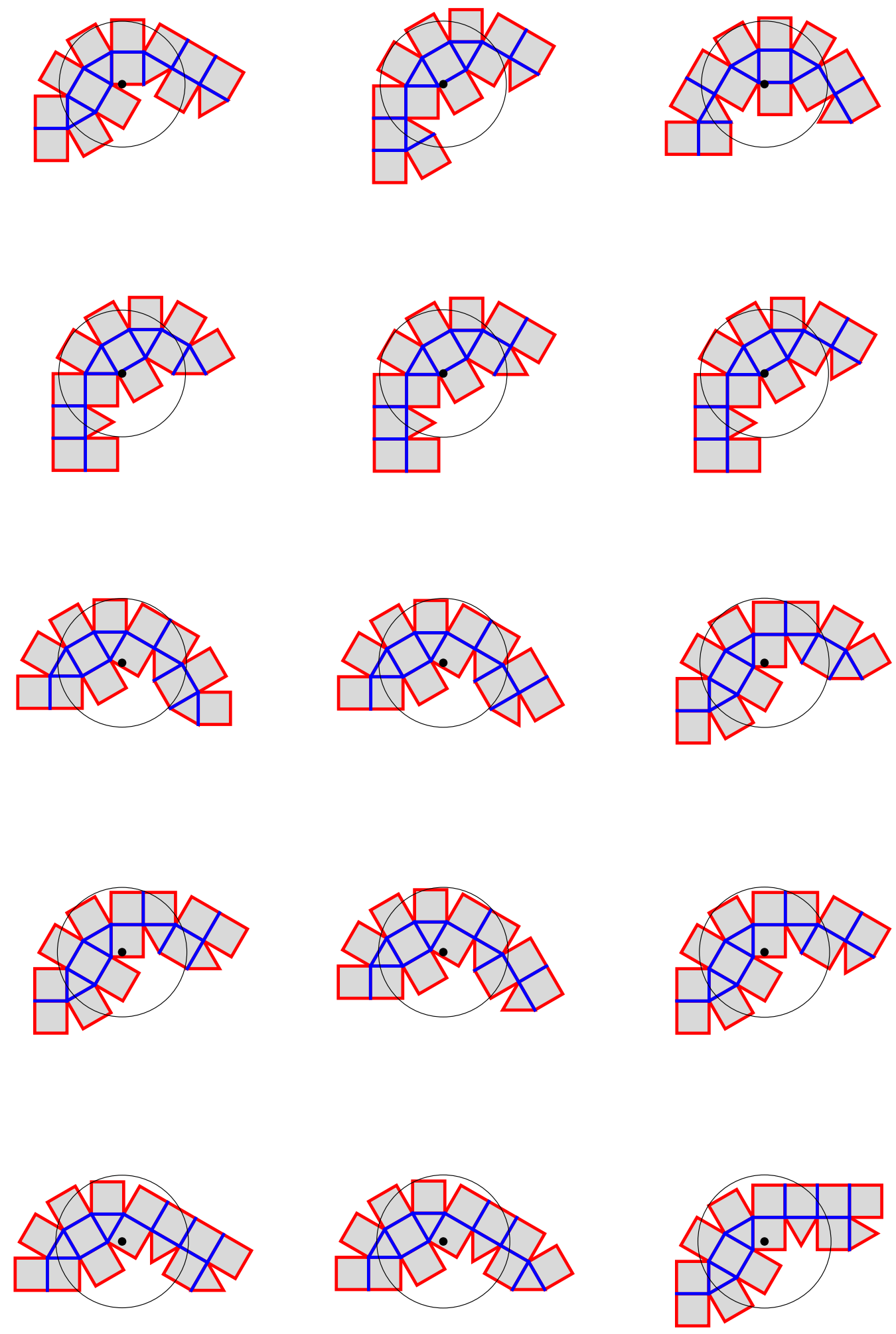

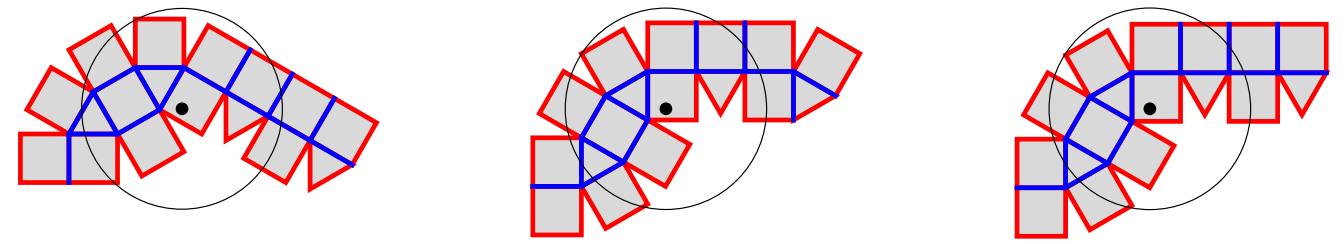

$\begin{array}{lll}\text { Ranked radii of gyration: } & \\ 1 . & 1.0022 & 1.00676 \\ 1.00676 & 1.00783 & 1.00938 \\ 1.0097 & 1.01069 & 1.01079 \\ 1.01103 & 1.0118 & 1.01329 \\ 1.01448 & 1.01464 & 1.01464 \\ 1.01692 & 1.01758 & 1.01758 \\ 1.01798 & 1.01806 & 1.01808 \\ 1.01827 & 1.01835 & 1.01863 \\ 1.0197 & 1.01974 & 1.02121 \\ 1.02205 & 1.02258 & 1.02299 \\ 1.02318 & 1.02423 & 1.02528 \\ 1.02528 & 1.02539 & 1.02563 \\ 1.02623 & 1.0268 & 1.02711 \\ 1.02918 & 1.03031 & 1.03053 \\ 1.03067 & 1.03181 & 1.03197 \\ 1.03422 & 1.03695 & 1.03879 \\ 1.03926 & 1.04016 & 1.04475 \\ 1.0449 & 1.04602 & 1.04643 \\ 1.05046 & 1.05083 & 1.05202 \\ 1.05226 & 1.05642 & 1.05788 \\ 1.05914 & 1.06532 & 1.07085 \\ 1.09213 & 1.0923 & 1.09473 \\ 1.09475 & 1.09793 & 1.09801 \\ 1.09822 & 1.10071 & 1.10082 \\ 1.10395 & 1.10635 & 1.10642 \\ 1.11143 & 1.11748 & 1.12308 \\ 1.128 & 1.13067 & 1.13327 \\ 1.13584 & 1.13688 & 1.14206 \\ 1.16303 & 1.16738 & 1.1678 \\ 1.16877 & 1.17228 & 1.17353\end{array}$

Optimal net of the Small Rhombicuboctahedron without top 9 faces with minimum radius of gyration: 


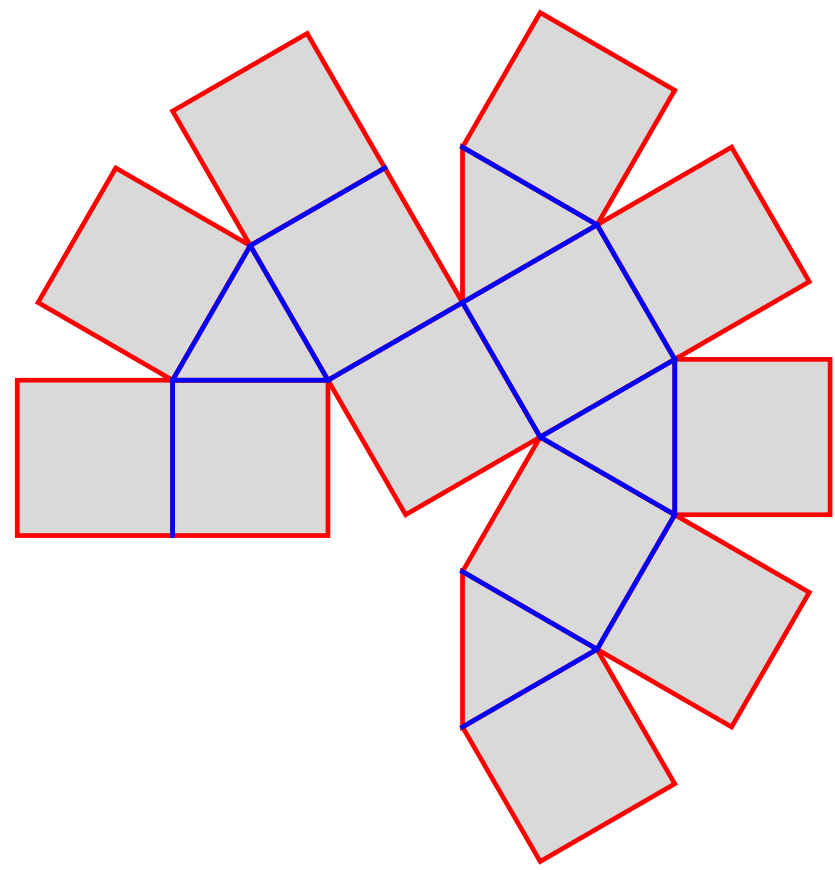

Plot of radius of gyration vs. rank of optimal nets:

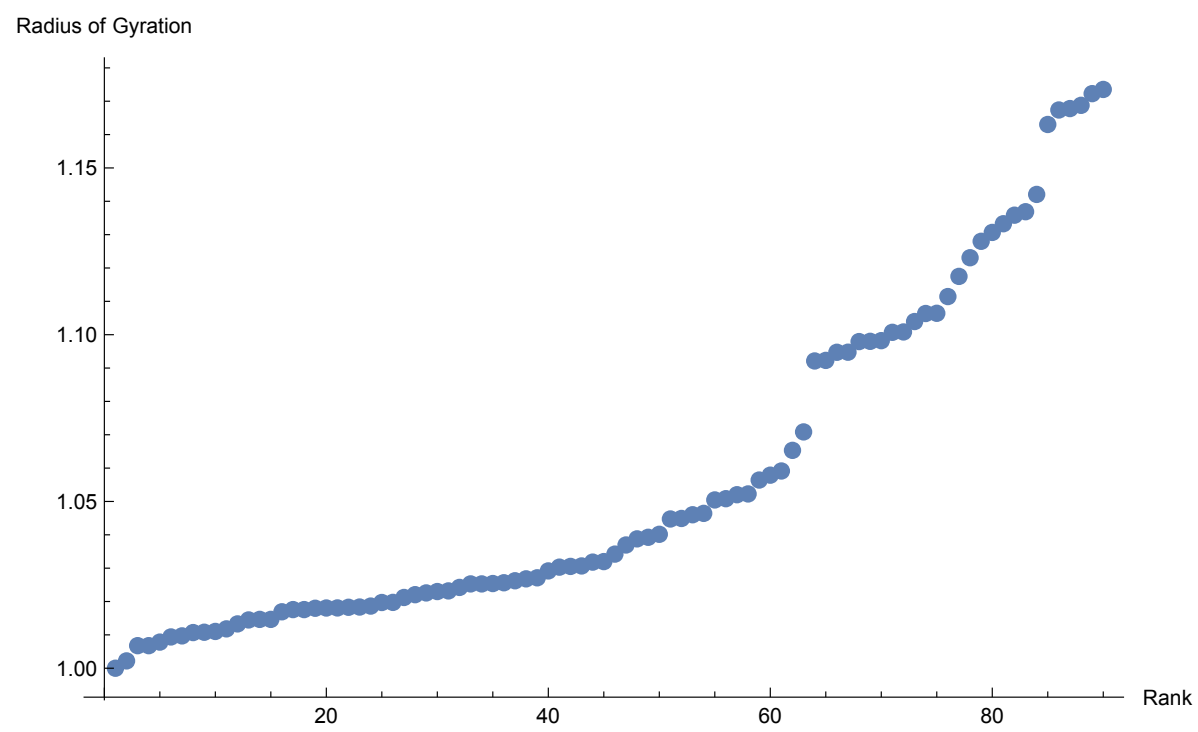


